\title{
تقييم كفاءة شبكة النقل بين المراكز الحضرية في محافظة بغداد
}

أ.د عبدالرحمن جري مردان الحويدر

جامعة البصرة / كلية التربية للعلوم الانسانية

rahmanja19@yahoo.com
الباحث بشار جبر علي

جامعة البصرة / كلية التربية للعلوم الانسانية basharj154@gmail.com

(مُلَخََّصُ البَحثث)

يهدف البحث لبيان كفاءة شبكة الطرق الرابطة بين المراكز الحضرية في محافظة بغداد، من خلال التقييم الكمي لواقع شبكة الطرق، لتقديم الخدمات المطلوبة بأنسيابية وكفاءة عالة لمستخدميها، كونها تمثل شرايين الحركة بين المراكز الحضرية، لذ اقتضى استخدام عدد من المقاييس والمؤشرات الاحصائية المعتمدة لتعطي مؤشراً عن كفاءة الطرق ولتحقق

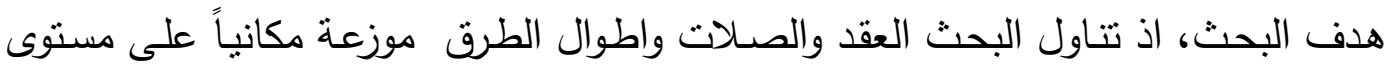
محافظة بغداد، اذ ترتبط كل عقدة بوصلات مباشرة وغير مباشرة وبأطوال مختلفة. وطبقت الدراسة المؤشرات الكمية في قياس كفاءة شبكة الطرق الرابطة بين العقد، فقد توصل مقياس كثافة الطرق بالنسبة للسكان وللمسـاحة الى وجود نباين في درجة الكثافة بين الوحدات

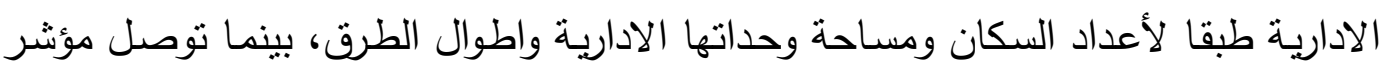

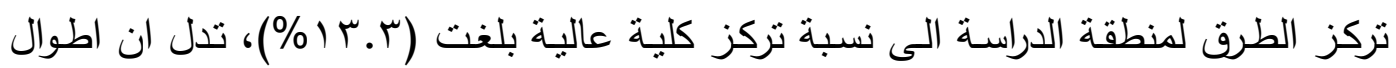

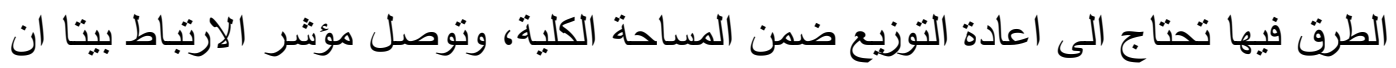

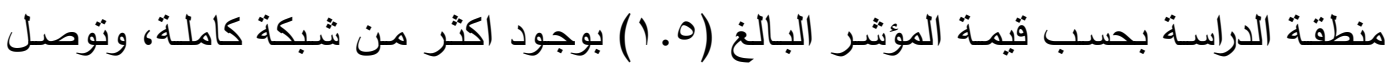

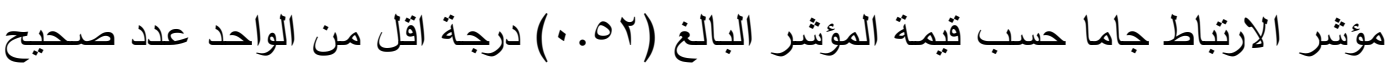

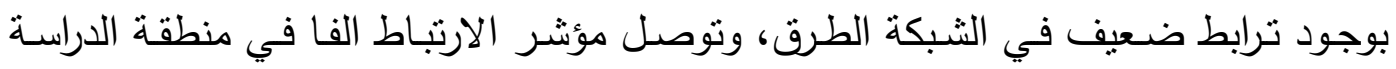

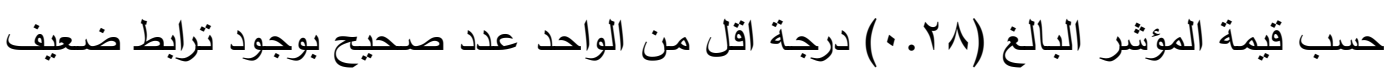

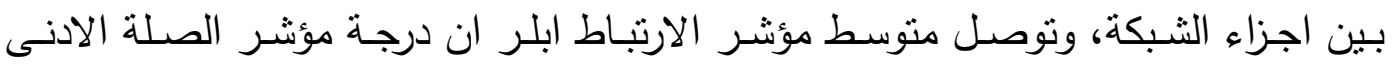

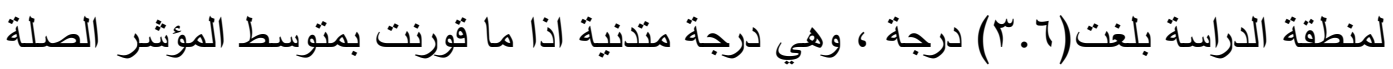

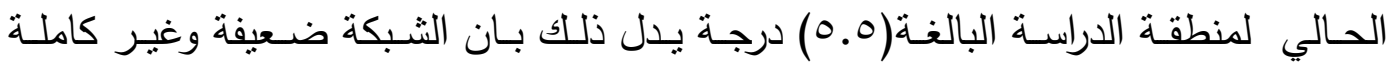

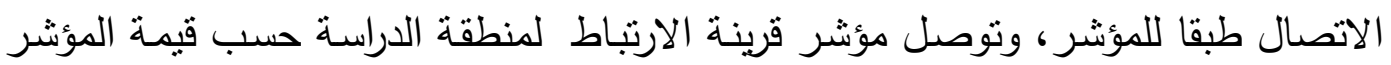

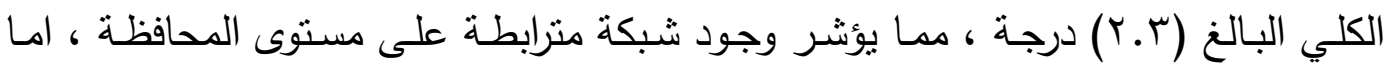

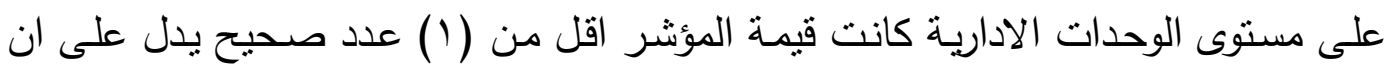

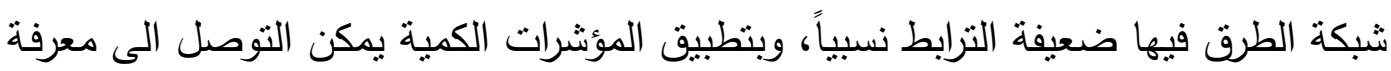
قوة وضعف شبكة الطرق، واختيار الحلول الناجحة لها. 
يعد النقل أحد مرتكزات البنى التحتيـة التي يعتمد عليها نجاح أي نشـاط اقتصادي ،

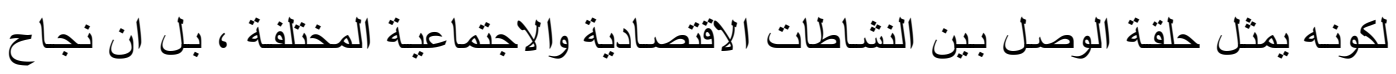
الكثير من هذه النشاطات يتوقف على مقدار كفاءة منظومة شبكة النقل سواء داخل الحيز الحضري او بين المراكز الحضرية . لمنئ

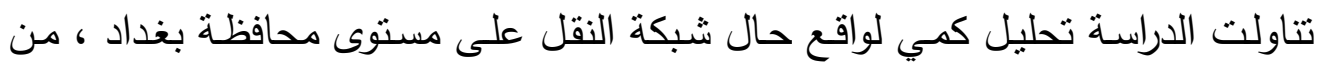

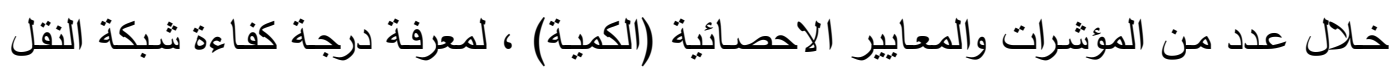

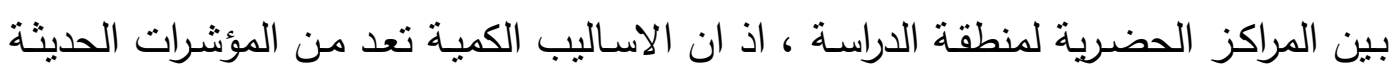

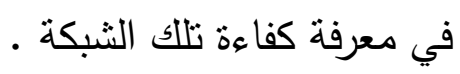
هدف البحث: يهدف البحث الى تقييم كفاءة شبكة النقل بين المراكز الحضرية في محافظة

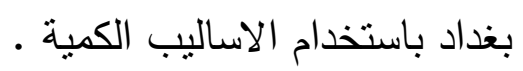
مشـكلة البحـث: مـا مدى وجود شبكة طرق ذات ترابط كفوء بين المراكز الحضرية في

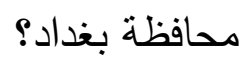
فرضية البحث: يفترض البحث عدم وجود شبكة طرق منرابطة وكفؤة وفق المؤشرات الكية في محافظة بغداد. حدود البحث: يتحدد البحث مكانباً بمحافظة بغداد باستثناء مدينة بغداد والبلديات التابعة لها،

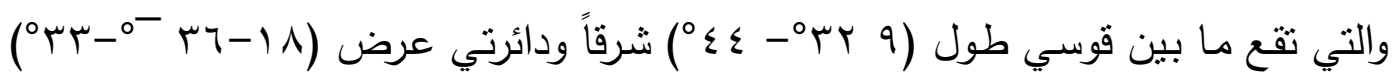

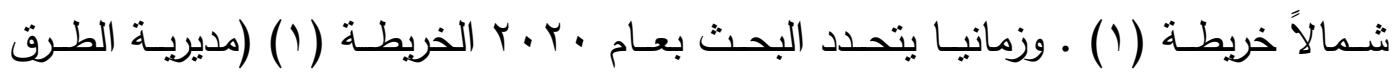

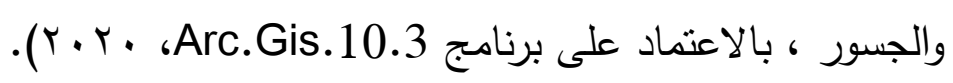

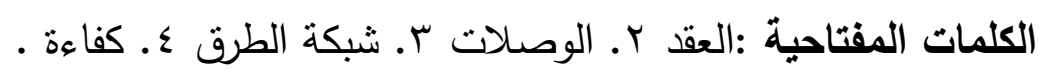

\section{اولاً : مقاييس كفاءة الطرق: الطفاه:}

تمثل شبكة النقل العلاقة الحقيقية بين الانسان وبيئته ، لاسيما اذا ارتبط ذلك

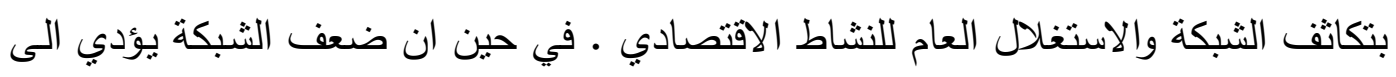

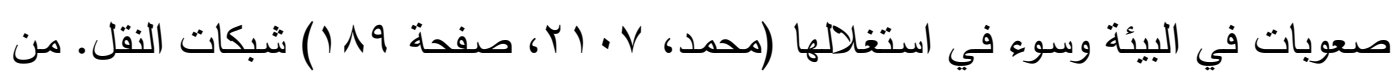
اجل الوصول الى نتائج اكثر دقة يتطلب استخدام بعض الاساليب الكمية التي تكون نتائجها

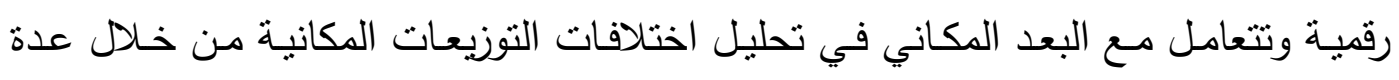
مؤشرات، منها مؤشر الكثافة ومؤشر اعادة التوزيع ومؤشر معامل التركيز (ابراهيم، دراسات

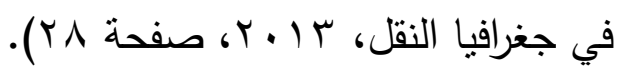




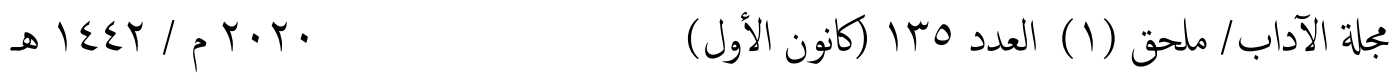

الخريطة (1)

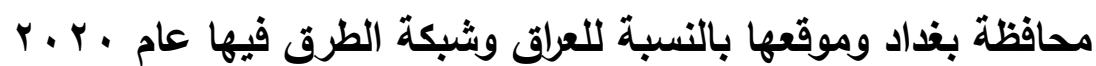

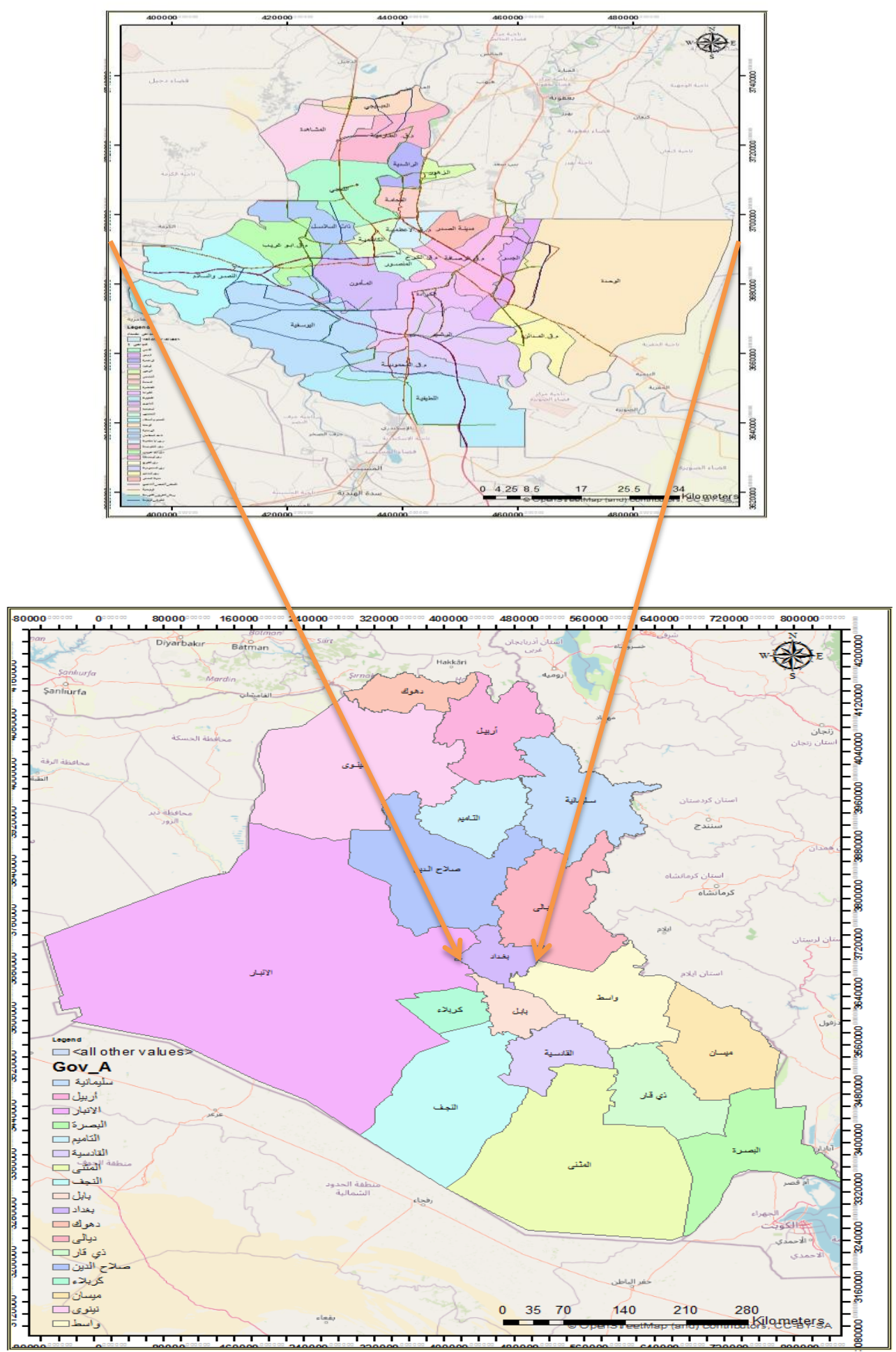


هناك علاقة بين اطوال الطرق من جهة وبين عدد السكان والمساحة واعداد السيارات

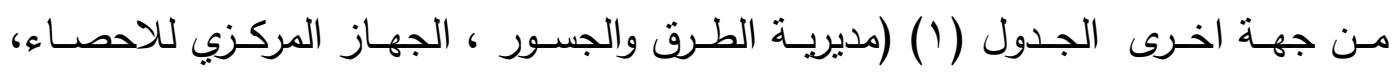

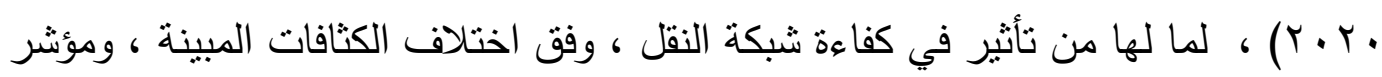

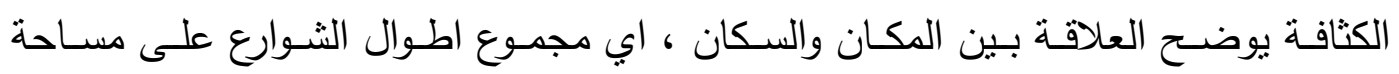

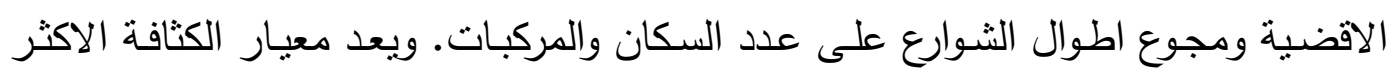
شيوعا لأغراض الدراسة المقارنة. أ. كثافة الطرق بالنسبة لأعداد السكان:

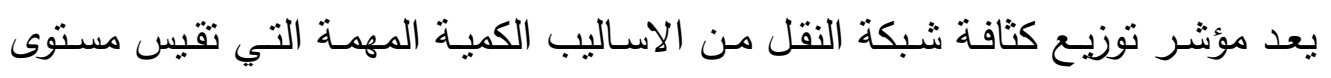

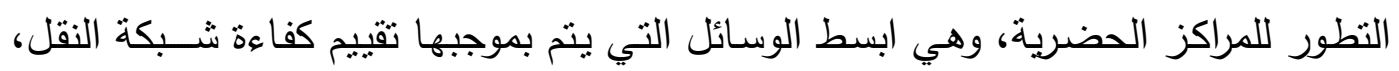

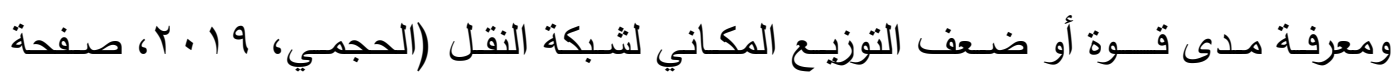

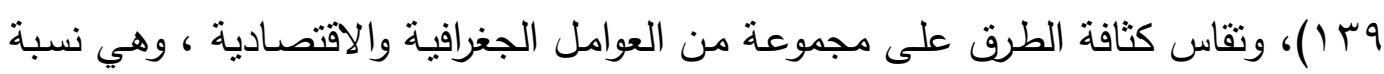

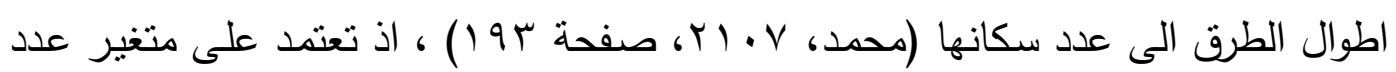

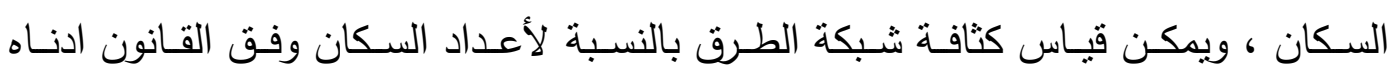

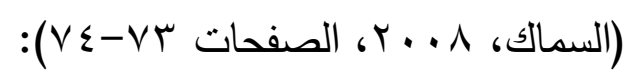

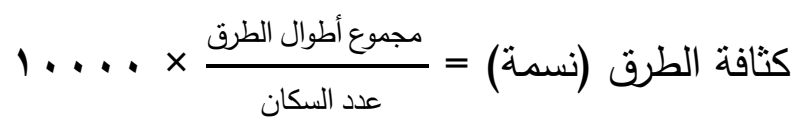

الجدول (1) التوزيع المكاني لكثافة الطرق طبقاً لأعداد السكان في المراكز الحضرية

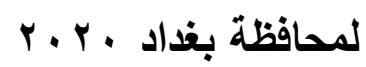

\begin{tabular}{|c|c|c|c|c|c|c|}
\hline 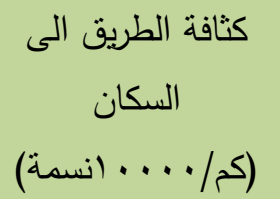 & النسبة & لعام السكانية & النسبة \% & 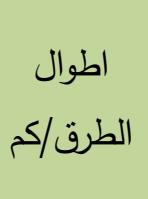 & المراكز الحضرية & ت \\
\hline T.T & 1.0 & $\varepsilon r, T \vee \wedge$ & 1.1 & 11.0 & ناحية الراشدية & 1 \\
\hline.$r$ & Tr.t & $\tau \leqslant r, 00 \wedge$ & 1.7 & 17 & ناحية الفحامة & r \\
\hline.$\varepsilon$ & $V .0$ & $(Y) 1, .99$ & $\cdot .1$ & A. 1 & ناحية الزهور & r \\
\hline r.T & 9.9 & $\lceil\wedge \Lambda, \Gamma \leq \varepsilon$ & $\vee . \varepsilon$ & $V \leqslant .0$ & ناحية ذات السلاسل & $\varepsilon$ \\
\hline $1 . \varepsilon$ & 0.9 & $|V|, V \mid V$ & Y.乏 & $r \varepsilon$ & ناحية التاجي & 0 \\
\hline r.r & $\varepsilon . Y$ & $I Y Y, T \cdot V$ & r.A & r^.० & م. ق. الرصافة & 7 \\
\hline$r .7$ & r.9 & A.,9ז4 & r.l & $r \cdot . \Lambda$ & م. ق. المدائن & v \\
\hline r. & $0 . V$ & $170, \wedge r q$ & $r .7$ & Tr & ناحية الجسر & $\wedge$ \\
\hline$\varepsilon . \wedge$ & V.r & YIr,^৭^ & $1 . r$ & $1 \cdot r$ & ناحية الوحدة & 9 \\
\hline 0.0 & 0.7 & $17 ., 970$ & $\Lambda . \Lambda$ & $\wedge \wedge$ & م. ق. المحمودية & 1. \\
\hline
\end{tabular}


$\Delta \sum \sum \varepsilon / \beta T \cdot T$.

مجلة الآداب/ ملحق (1) العدد هبا (كانون الأول)

\begin{tabular}{|c|c|c|c|c|c|c|}
\hline $11 . r$ & $\varepsilon . V$ & $1 T V, \cdot \leq 7$ & 10.1 & 104.0 & ناحية اليوسفية & 11 \\
\hline 9.1 & r.o & $1 \cdot Y, Y Y T$ & $9 . r$ & qr.V & ناحية اللطيفية & IT \\
\hline 11.9 & r. 9 & АT,VTV & 1. & $1 \cdots$ & ناحية الرشيد & س \\
\hline 0.0 & $0 . \varepsilon$ & $100,10 \mathrm{r}$ & $\Lambda .7$ & $\wedge \uparrow$ & م. ق. ابو غريب & $1 \varepsilon$ \\
\hline r.r & 0.1 & $\mid T V, Y 17$ & r.^ & ऍ^.દ & النصر والسـلم & 10 \\
\hline 7 & r. & $q 7, r q r$ & 0.1 & ov.V & م. ق. الطارمية & 17 \\
\hline $10 . \varepsilon$ & $1 . r$ & & $0 . Y$ & 01.9 & ناحية المشاهدة & iv \\
\hline A.l & .0 & $10,1 \leq 9$ & $1 . r$ & מ.t & ناحية العبايجي & 11 \\
\hline $9 \leq .1$ & $1 \ldots$ & r^৭৭^VV & $1 \ldots$ & $1 . .4 .9$ & \multicolumn{2}{|c|}{ المجموع الاجمالي } \\
\hline
\end{tabular}

تم تقسيم الفئات وفق القانون التالي :

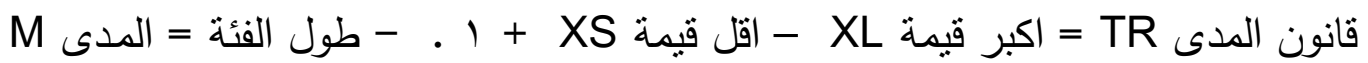

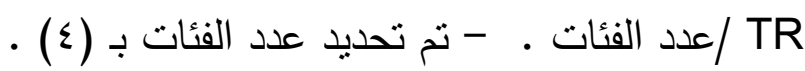

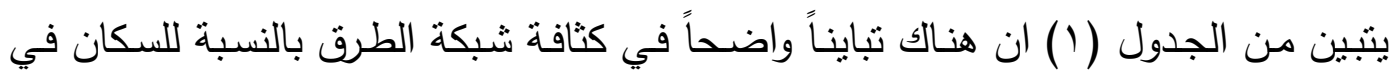

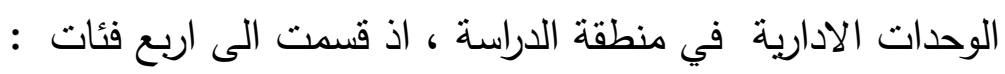

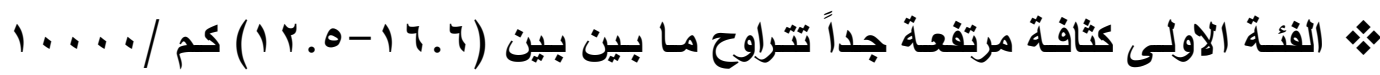
نسمة : 20 - n

تحظى ناحية المشـاهدة بالمرتبة الاولى كأعلى مستوى لكثافة الطرق بالنسبة لأعداد

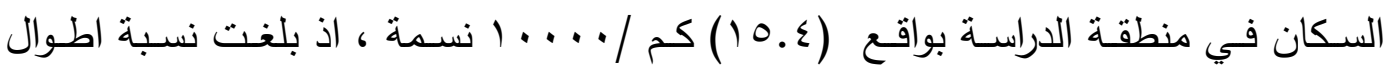
الطرق للناحية (r.0\%) ، اي انها في المرتبة العاشرة من مجموع اطوال الطرق في منطقة

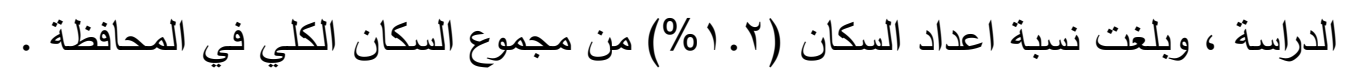

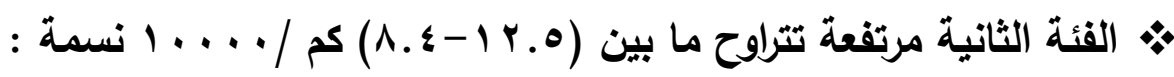
تتمثل هذه الفئة في ناحية الرشيد التي سجلت في المرتبة الثنانية على مستوى منطقة

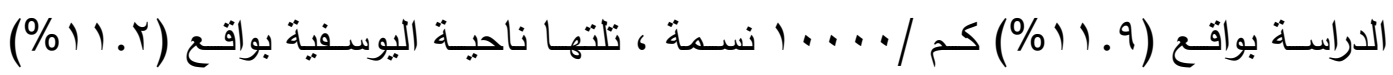

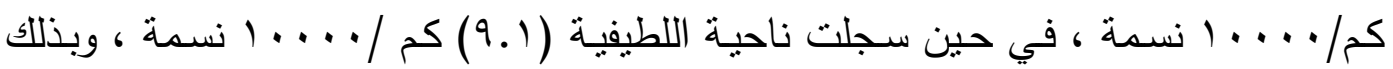
تكون ضمن المرتبة الاخيرة في هذه الفئة .

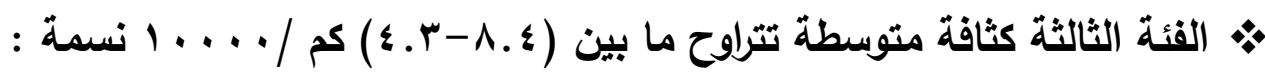

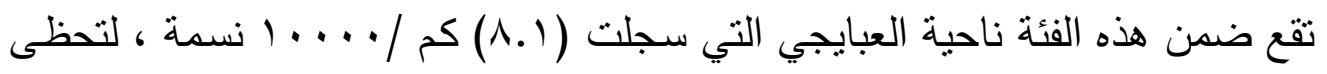

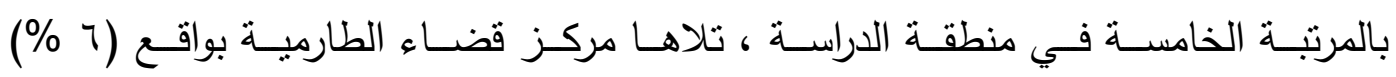

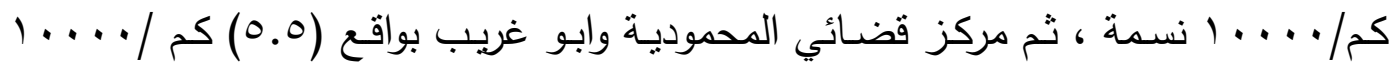

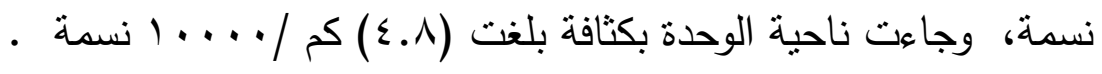




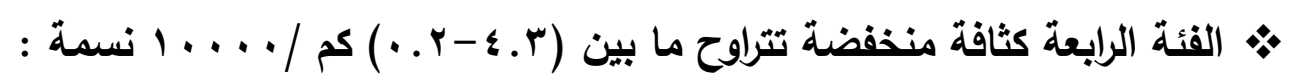

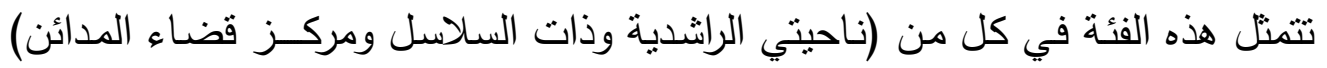

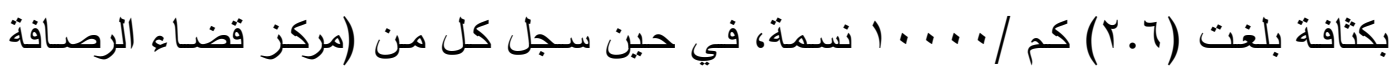

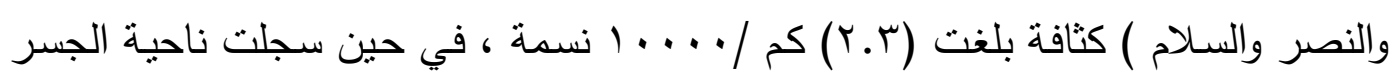

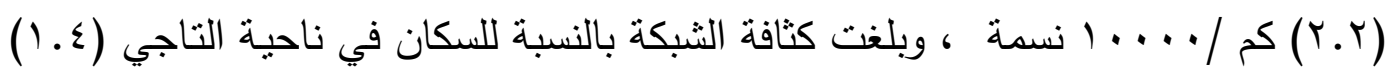

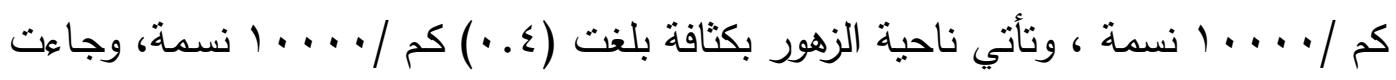

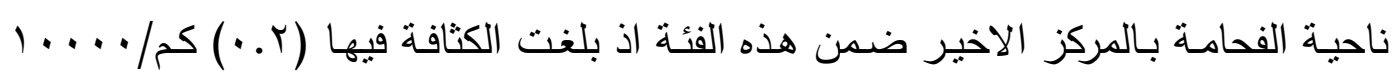

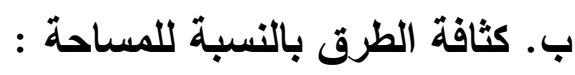
يعد مؤشـر توزيـع كثافة شبكة النقل بالنسبة للمسـاحة احد اهم المقاييس التي تقيس

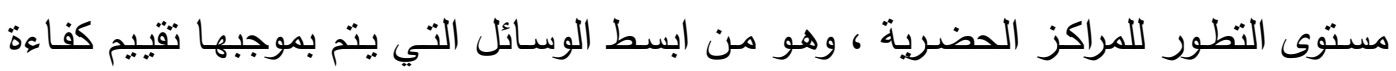

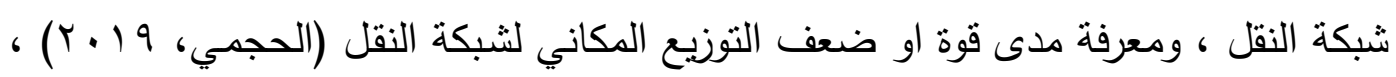

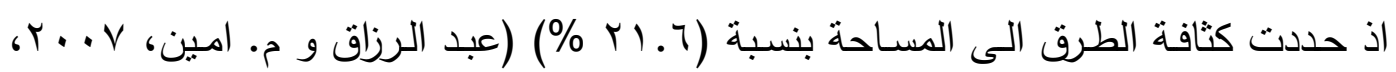

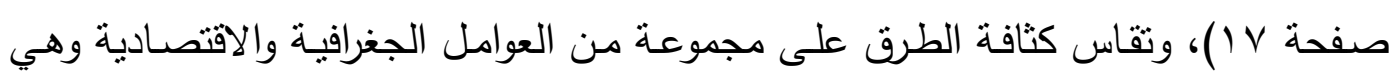

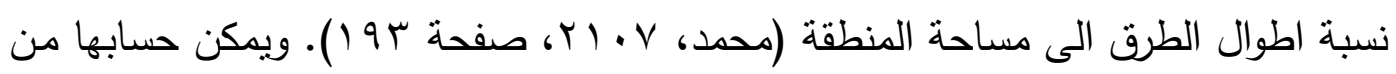

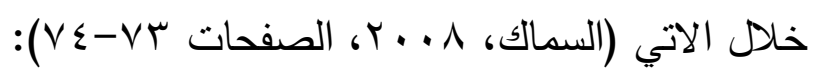

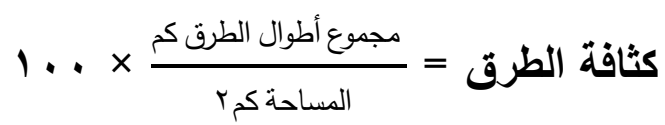

تم تقسيم كثافة الطرق بالنسبة للمساحة حسب الوحدات الادارية الى اربع فئات الجدول

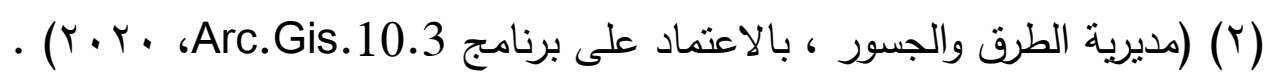

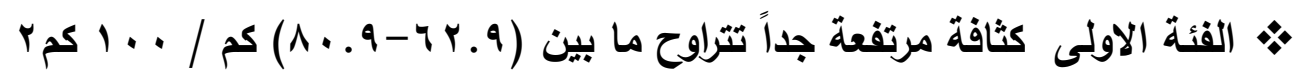

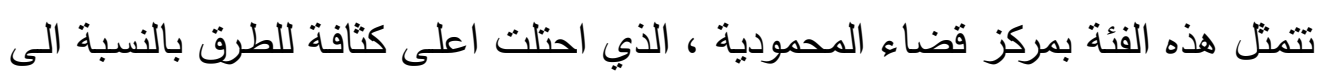

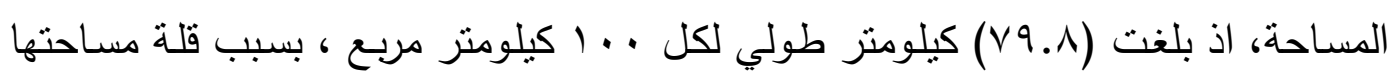

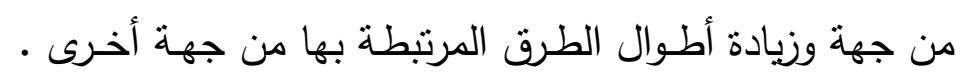

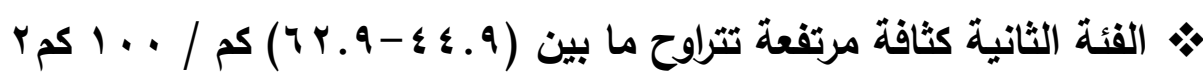

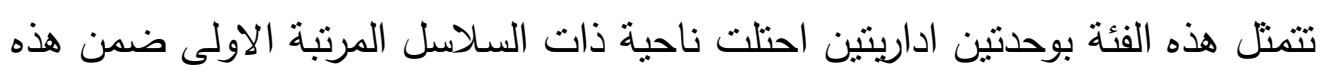

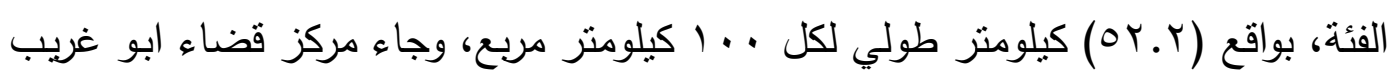

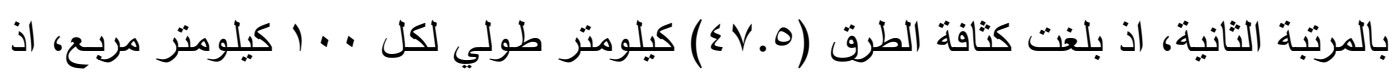
امتازا بوجود طرق نقل جيدة وتوفر اراضي صالحة للزراعة تساعد على توطن السكان. 


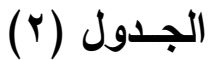

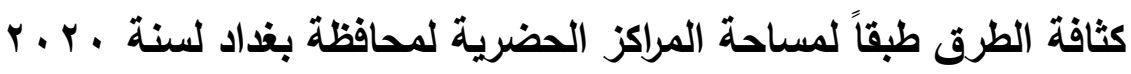

\begin{tabular}{|c|c|c|c|c|c|c|}
\hline كثافة الطرق كمالمساحة & النسبة & كمباحة | المسا & النسبة\%\% & الطرق/ اطو كم & المراكز الحضرية & $ت$ \\
\hline 17.7 & 1.0 & $79 . \varepsilon$ & 1.1 & 11.0 & ناحية الراشدية & 1 \\
\hline rq.1 & $1 . r$ & $0 \leqslant .9$ & 1.7 & 17 & ناحية الفحامة & r \\
\hline rr &. .1 & r.1 &. .1 & A.) & ناحية الزهور & r \\
\hline OY.Y & r.l & $1 \leq Y .7$ & $V . \varepsilon$ & $V \varepsilon .0$ & ناحية ذات السلاسل & $\varepsilon$ \\
\hline $1 \cdot$ & 0.1 & r苗.q & Y. $\varepsilon$ & $r \varepsilon$ & ناحية التاجي & 0 \\
\hline $1 \varepsilon$ & $\varepsilon . \varepsilon$ & $r \cdot r . \tau$ & Y.A & rᄉ. 0 & م. ق. الرصافة & 7 \\
\hline $1 \leq . V$ & r. & $1 \leq 1.1$ & r. 1 & $r \cdot . \Lambda$ & م. ق. المدائن & V \\
\hline س - س & r.r & $1 \cdot \Lambda . V$ & r.7 & (ب & ناحية الجسر & $\wedge$ \\
\hline 1.9 & ro & 1101.1 & 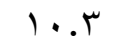 & $1 \cdot r$ & ناحية الوحدة & 9 \\
\hline$\vee 9.1$ & Y. $\varepsilon$ & س. • & ᄉ.^ & $\wedge \wedge$ & م. ق. المحمودية & 1 . \\
\hline rq.9 & 11.1 & $01 \leqslant$ & 10.1 & $10 \% .0$ & ناحية اليوسفية & 11 \\
\hline 19.8 & $1 \cdot .1$ & $\varepsilon 79 . V$ & $9 . r$ & QY.V & ناحية اللطبفية & Ir \\
\hline rᄉ.r & $V .7$ & 1. 1. & $1 \cdot$ & $1 \cdots$ & ناحية الرشيد & r \\
\hline$\leqslant V .0$ & r. & 111 & A. 7 & $\wedge \neg$ & م.ق. ابو غريب & $1 \leq$ \\
\hline $1 \cdot . r$ & A. 1 & rvV.r & r.A & H人.乏 & النصر والسلام & 10 \\
\hline V.V & r.v & $|V| . \varepsilon$ & 0.1 & $O V . V$ & م. ق. الطارمية & 17 \\
\hline ro & $\leqslant .0$ & $Y \cdot V . V$ & $0 . r$ & 01.9 & ناحية المشاهدة & iv \\
\hline 14.9 & r & 90.1 & $1 . r$ & IT.T & ناحية العبايجي & 11 \\
\hline Y 1.7 & $1 \ldots$ & 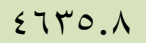 & $1 \ldots$ & $1 . .4 .9$ & \multicolumn{2}{|c|}{ المجموع الاجمالي } \\
\hline
\end{tabular}

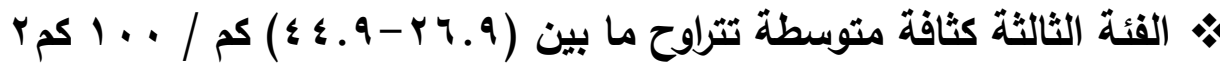

تشمل هذه الفئة خمس وحدات اداريـة جاء مركز قضـاء الطارمية بمقدمتها ، اذ سجل

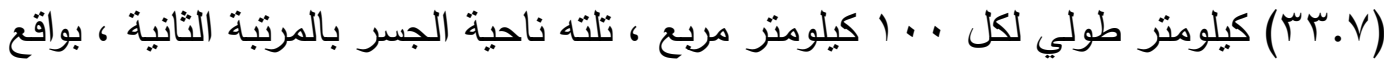

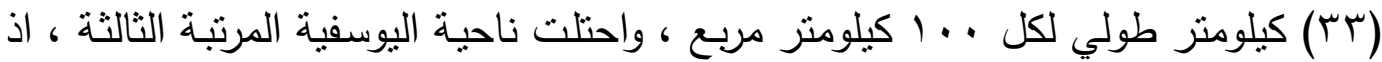

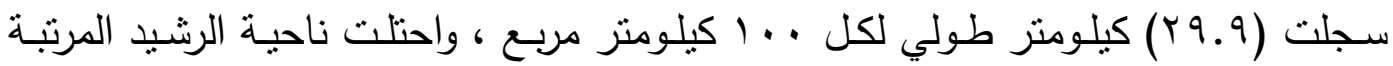

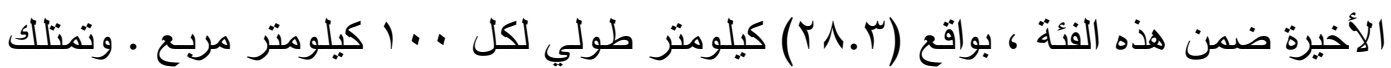
الوحدات الادارية ضمن هذه الفئة مقومات متعددة اهمها صلاحية اراضيها الزراعية وامتداد الاده 


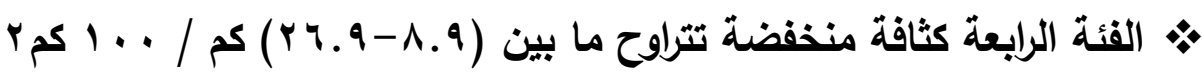

تقع ضمن هذه الفئة عشر وحدات اداريـة تتمثل بكل من (ناحية المشـاهدة ، ناحية الزهور ، ناحية اللطيفية ، ناحية الراثدية ، مركز قضاء المدائن ، مركز قضاء الرصافة ،

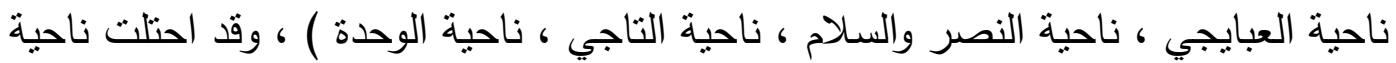

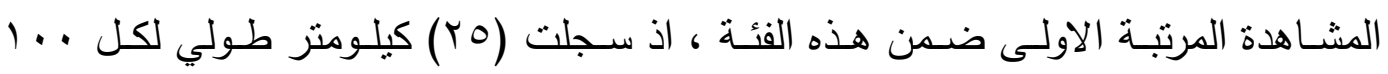

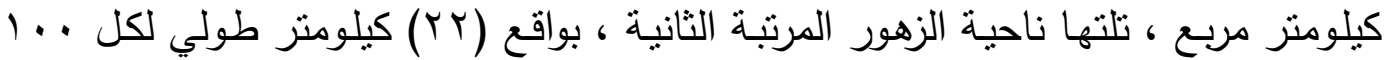

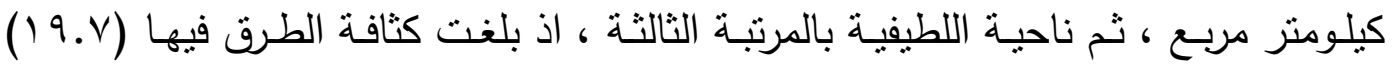
كيلومتر طولي لكل .ا كيلومتر مربع · في حين جاءت ناحية الوحدة المرنبة الاخيرة

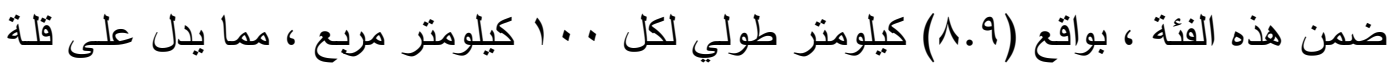
اطوال الطرق فيها وحاجتها الى زيادة اطوال الطرق بما يتتاسب مع مساحتها الكلية .

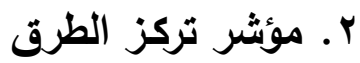
يعد مؤشر تركز الطرق من المؤشرات المهمة ، التي يمكن من خلالها معرفة مدى لفى التشتت أو التركز لأطوال الطرق ، من خلال العلاقة العددية بين اطوال الطرق والمساحة

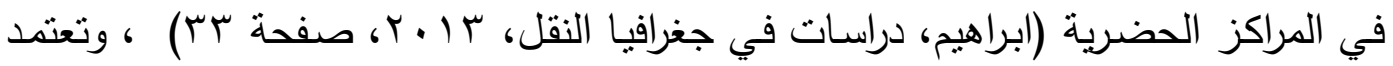
نسبة التوزيع على القيمة الرقمية ، فكلما ارتفعت القيمة الرقمية الكلية للمؤشر تدل على وجود

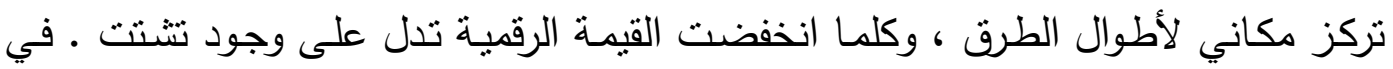

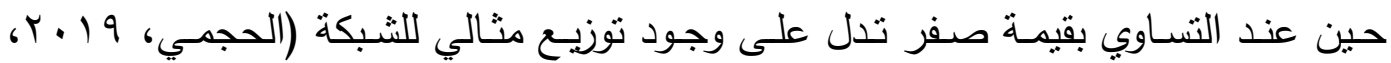

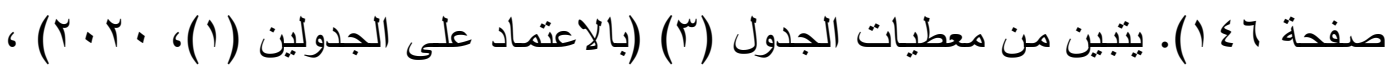

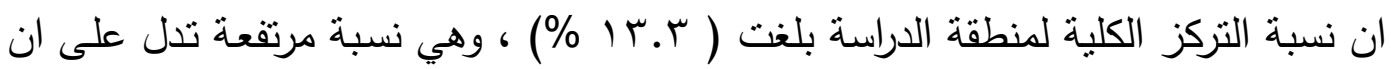

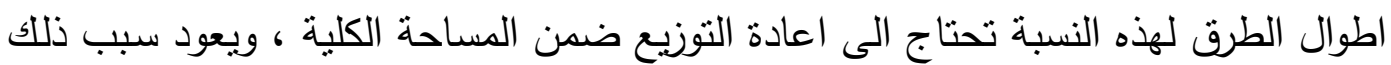
الى التفاوت في حجم المساحات ، والسكان في المراكز الحضرية . شكل مؤشر التركز بالإثـارة الموجبة على مستوى النواحي نسب متفاوتة من مركز

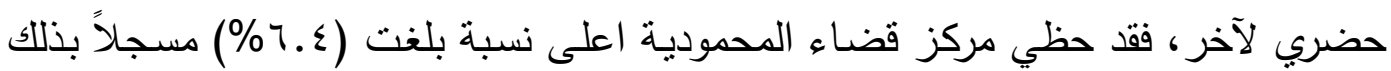

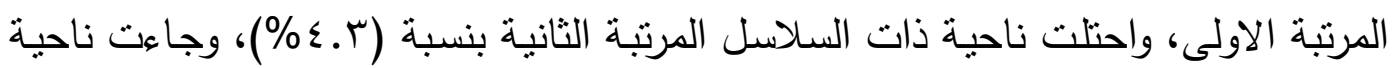
اليوسفية بالمرتبـة الثالثة بنسبة بلغت (؟.؛ \%) ، وجاءت بالمرتبـة الاخيرة ناحيـة الفحامـة

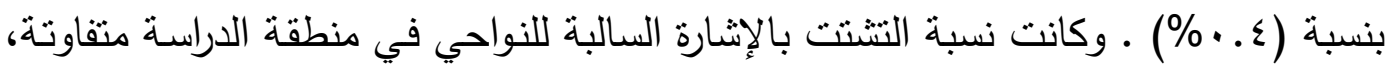

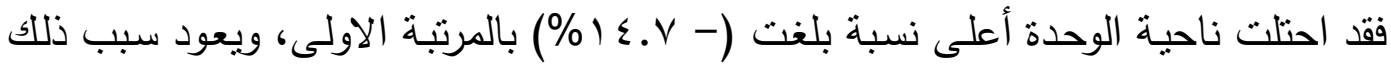

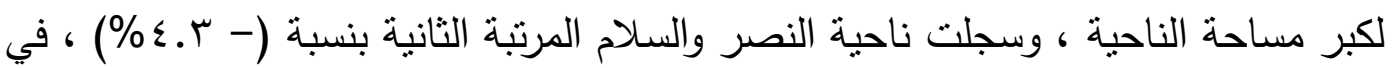

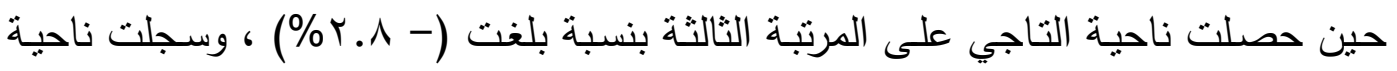


الراشدية المرتبـة الاخيرة باقل نسبة بلغت (- ع. • \%\%) ـ كما يتضح من الجدول (r) بان

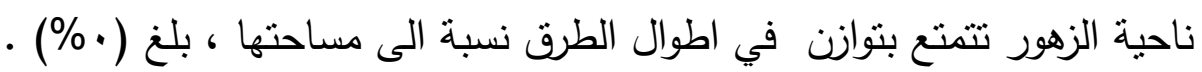
كما يتبين من خلال ملاحظة نتائج مؤشر التركز والتشتت في التوزيع الجغرافي لأطوال

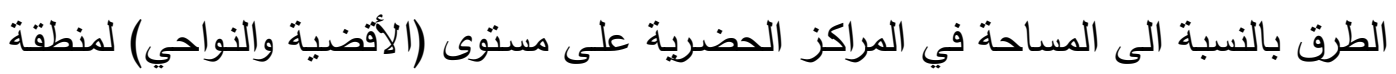

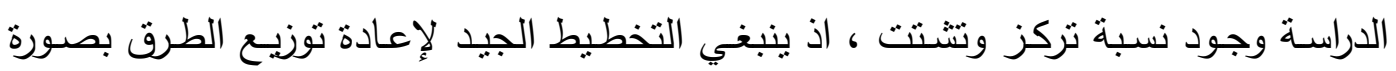

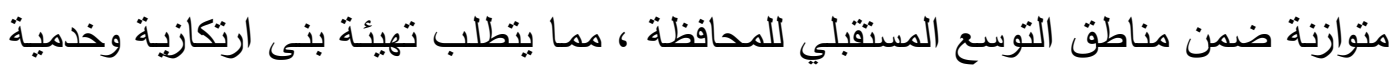
ومن اهما شبكة الطرق .

\section{الجـدول (r)}

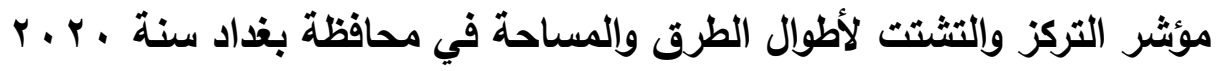

\begin{tabular}{|c|c|c|c|c|c|c|}
\hline الفرق النسبي بين & النسبة\% & المساحة & النسبة\% & الطرق/كم الطوال & المراكز الحضرية & $ت$ \\
\hline$\cdot . \varepsilon-$ & 1.0 & 79.5 & 1.1 & 11.0 & ناحية الراشدية & 1 \\
\hline$\cdot . \varepsilon+$ & $1 . r$ & $0 \leqslant .9$ & 1.7 & 17 & ناحية الفحامة & r \\
\hline$\cdot$ & $\cdot \wedge$ & 4.1 & $\cdot . \wedge$ & A.1 & ناحية الزهور & r \\
\hline$\varepsilon . r+$ & r.l & $1 \leq Y .7$ & V.乏 & $V \leqslant .0$ & ناحية ذات السلاسل & $\varepsilon$ \\
\hline r.^ - & $0 . r$ & rr人.q & T.乏 & $r \leq$ & ناحية التاجي & 0 \\
\hline $1.7-$ & $\varepsilon . \varepsilon$ & $r \cdot T . \tau$ & r.A & r^.0 & م. ق. الرصافة & 7 \\
\hline $1-$ & r.l & $1 \leq 1.1$ & r.l & $r \cdot . \Lambda$ & م. ق. المدائن & v \\
\hline $1 . r+$ & t.r & $1 \cdot \Lambda . V$ & T.7 & tra & ناحية الجسر & $\wedge$ \\
\hline $1 \leqslant . V-$ & ro & 1101.1 & $1 . r$ & $1 . r$ & ناحية الوحدة & 9 \\
\hline 7. $\Sigma+$ & Y.乏 & 11. & $\Lambda . \Lambda$ & $\wedge \wedge$ & م. ق. المحمودية & 1. \\
\hline$\varepsilon . r+$ & 11.1 & $01 \leq$ & $10 . r$ & 104.0 & ناحية اليوسفية & 11 \\
\hline$\cdot .9-$ & $1 \cdot .1$ & $\leq 79 . V$ & 9.1 & 9 QY.V & ناحية اللطيفية & ir \\
\hline Y. $\Sigma+$ & $\vee .7$ & ror.A & 1. & $1 \ldots$ & ناحية الرشيد & M \\
\hline$\varepsilon . V+$ & r.9 & 111 & 1.7 & $\wedge 7$ & م. ق. ابو غريب & $1 \varepsilon$ \\
\hline$\varepsilon . r-$ & A.) & rVV.r & r.ی & ґ৯.乏 & النصر والسلام & 10 \\
\hline r.l + & r.V & $|V| . \varepsilon$ & 0.1 & ov.V & م. ق. الطارمية & 17 \\
\hline$. V+$ & $\varepsilon .0$ & $r \cdot V \cdot V$ & $0 . r$ & 01.9 & ناحية المشاهدة & iv \\
\hline$\cdot . \wedge-$ & r & 90.1 & $1 . r$ & Ir.r & ناحية العبايجي & 11 \\
\hline $\begin{array}{l}\text { r..0+ } \\
\text { r..0- }\end{array}$ & $1 \ldots$ & $\leqslant 7 r 0.1$ & $1 \ldots$ & 1 ..r.q & وع الاجمالي & \\
\hline
\end{tabular}

تم تطبيق معادلة نسبة التركز للمساحة الكلية = 1/ مجموع (س) - (ص) 


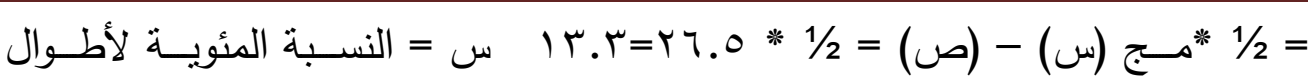
الطرق الى مجموع اطوال الطرق الكلية في منطقة الدراسة

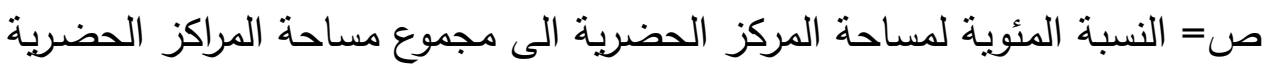
الكلية في منطقة الدراسة ، مـج = مجموع الفرق الموجب بين هذه النسب باعتماد الاشتارات الموجبة اي مجموع القيم الموجبة وإهمال الاثشارات السالبة. (ابراهيم ع.،

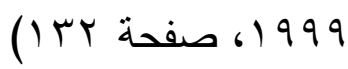

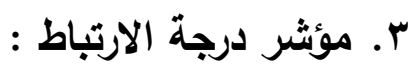

عند رسم خارطة لثبكة الطرق في منطقة الدراسة ، على انها مجموعة من العقد ترتبط فيما بينها بمجموعة من الوصلات المتصلة ، فان المقياس الاساسي لوصف شيكة شبكة الطرق، وتطورها ضمن منطقة الدراسة هو درجة الارتباط لكل ازواج العقد الجدول(ع) (بالاعتماد

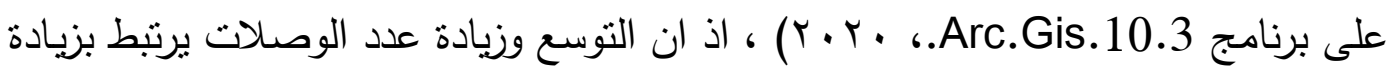

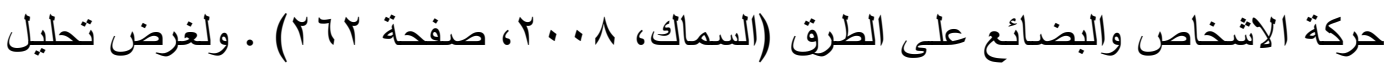
وتقييم كفاءة شبكة النقل بشكل عام والطرق بشكل خاص ، لابد ان يتم تحويل خريطة شبكة الطرق الى شكل تظهر فيه الوصلات بشكل خطوط مستقيمة تتفادى الانعطاف بين العقد (Adams, p., Network Topologies and Virtual Place, Annals of the A. A. G., 1988, p. 88)

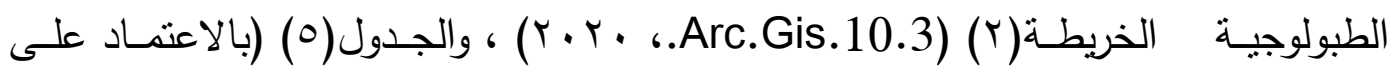

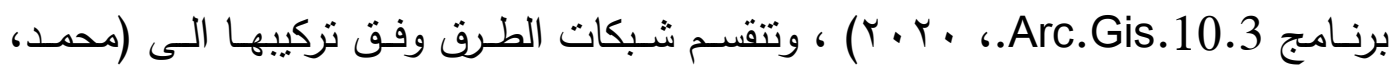

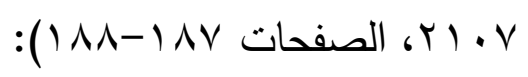

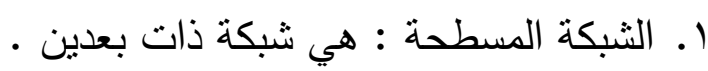

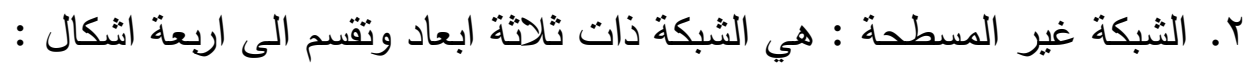

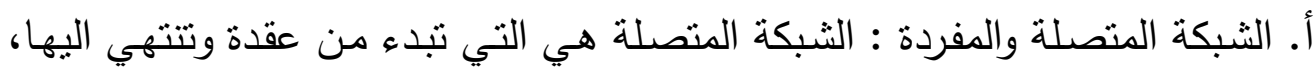
بينما الثبكة المفردة فهي التي تبدء من عقدة وتتنهي بأخرى .

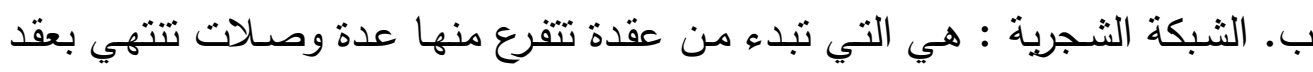

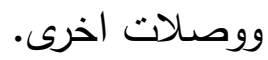

ج. الثبكة الدائرية : الثبكة التي تبدء من عقدة محددة وتتصل بالعقد الاخرى خلال وصلات تنتهي بالاولى.

د. الثبكة الخليات : هي التي تظم شبكة ضخمة تضم شبكات صغيرة . يتضح ان شبكة الطرق في منطقة الدراسة تميل الى نوع الثبكة الدائرية ، وقد اعتمد

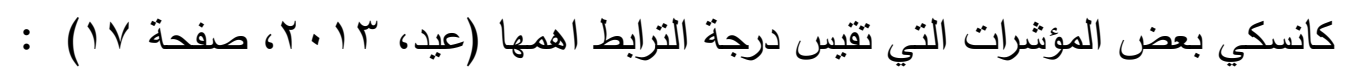


الجدول (ع r. r. مصفوفة العقد البينية لمحافظة بغداد سنة

\begin{tabular}{|c|c|c|c|c|c|c|c|c|c|c|c|c|c|c|c|c|c|c|}
\hline العباحبة & المشية & م.ق الطارمبة & والسـام & غ.ق. ابو & الرشية & اللطبية & |لناحية & م.ق المحمودية & ناحية & ناحية & | م.ق. & م.ق الرصضاء & |ناحية | & ناحية ذات & الزهية & |الفحامة & الراحية & المراكز الحضرية \\
\hline$r$ & $r$ & $r$ & $r$ & $r$ & 1 & $r$ & $r$ & $r$ & $r$ & 1 & $r$ & $\cdot$ & 1 & $r$ & $\cdot$ & $\cdot$ & & ناحية الر اشدية \\
\hline$r$ & 1 & 1 & $\mu$ & $r$ & 1 & $r$ & $r$ & $r$ & $r$ & 1 & $r$ & $\cdot$ & $\cdot$ & 1 & . & & . & ناحية الفحامة \\
\hline$r$ & r & $r$ & $r$ & $r$ & 1 & $r$ & $r$ & $r$ & $r$ & 1 & $r$ & . & 1 & $r$ & & . & . & ناحية الزهور \\
\hline$r$ & 1 & 1 & 1 & $\cdot$ & $r$ & $r$ & 1 & $r$ & $\varepsilon$ & $r$ & $r$ & $r$ & - & . & $r$ & 1 & $r$ & ناحية ذات السلاسل \\
\hline 1 & $\cdot$ & $\cdot$ & r & 1 & $r$ & $r$ & $r$ & $r$ & $r$ & T & $r$ & 1 & & . & 1 & $\cdot$ & 1 & ناحية التاجي \\
\hline$r$ & $r$ & $r$ & $r$ & $r$ & $\cdot$ & $r$ & 1 & 1 & 1 & $\cdot$ & 1 & & 1 & $r$ & $\cdot$ & $\cdot$ & $\cdot$ & م.ق. الرصافة \\
\hline 0 & $\varepsilon$ & $\varepsilon$ & $r$ & $r$ & $\cdot$ & $r$ & 1 & 1 & $\cdot$ & $\cdot$ & & 1 & $r$ & $r$ & $r$ & $r$ & $r$ & م.ق. المدائن \\
\hline$\varepsilon$ & $r$ & $r$ & $r$ & $r$ & . & $r$ & 1 & 1 & . & & - & . & $r$ & $r$ & 1 & 1 & 1 & ناحية الجسر \\
\hline 0 & $\varepsilon$ & $\varepsilon$ & $r$ & $r$ & 1 & $r$ & $r$ & $r$ & & $\cdot$ & . & 1 & $r$ & $\varepsilon$ & $r$ & $r$ & $r$ & ناحية الوحدة \\
\hline 0 & $\varepsilon$ & $\varepsilon$ & 1 & 1 & $\cdot$ & $\cdot$ & $\cdot$ & & $r$ & 1 & 1 & 1 & $r$ & $r$ & $r$ & $r$ & $r$ & م.ق. المحمودية \\
\hline$\varepsilon$ & $r$ & $r$ & . & $\cdot$ & . & . & & $\cdot$ & $r$ & 1 & 1 & 1 & $r$ & 1 & $r$ & $r$ & $r$ & ناحية اليوسفية \\
\hline 0 & $\varepsilon$ & $\varepsilon$ & 1 & 1 & 1 & & $\cdot$ & . & $r$ & $r$ & $r$ & $r$ & $r$ & $r$ & $r$ & $r$ & $r$ & ناحية اللطيفية \\
\hline$r$ & $r$ & $r$ & . & & 1 & 1 & $\cdot$ & 1 & $r$ & $r$ & $r$ & $r$ & 1 & $\cdot$ & $r$ & $r$ & $r$ & م.ق. ابو غريب \\
\hline$\varepsilon$ & $r$ & $r$ & & . & 1 & 1 & $\cdot$ & 1 & $r$ & $r$ & $r$ & $r$ & $r$ & 1 & $r$ & $r$ & $r$ & النصر والسلام \\
\hline$\cdot$ & $\cdot$ & & $r$ & $r$ & $r$ & $\varepsilon$ & $r$ & $\varepsilon$ & $\varepsilon$ & $r$ & $\varepsilon$ & $r$ & $\cdot$ & 1 & $r$ & 1 & $r$ & م.ق.الطارمية \\
\hline$\cdot$ & & $\cdot$ & $r$ & $r$ & $r$ & $\varepsilon$ & $r$ & $\varepsilon$ & $\varepsilon$ & $r$ & $\varepsilon$ & $r$ & $\cdot$ & 1 & $r$ & 1 & $r$ & ناحية المشاهدة \\
\hline & $\cdot$ & $\cdot$ & $\varepsilon$ & $r$ & $\varepsilon$ & 0 & $\varepsilon$ & 0 & 0 & $\varepsilon$ & 0 & $r$ & 1 & $r$ & $r$ & $r$ & $r$ & ناحية العبايجي \\
\hline or & rA & rA & T纟 & $r \wedge$ & YI & 49 & $r \varepsilon$ & $\mu$ & $\varepsilon 1$ & rT & $\Gamma \varepsilon$ & $r$. & ro & rq & rq & $r T$ & rq & المجموع \\
\hline 10 & Ir & IT & 11 & $V$ & $r$ & 14 & $\varepsilon$ & 9 & $1 \varepsilon$ & 7 & 1. & 1 & 0 & $\wedge$ & $\wedge$ & $r$ & $\wedge$ & الرتبة المرجحة \\
\hline
\end{tabular}




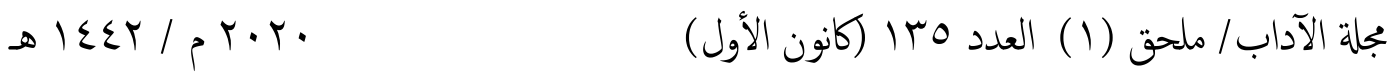

(ץ) الخريطة

r. r . . الثكل الطبولوجي للعقد والوصلات في محافظة بغداد لسنة

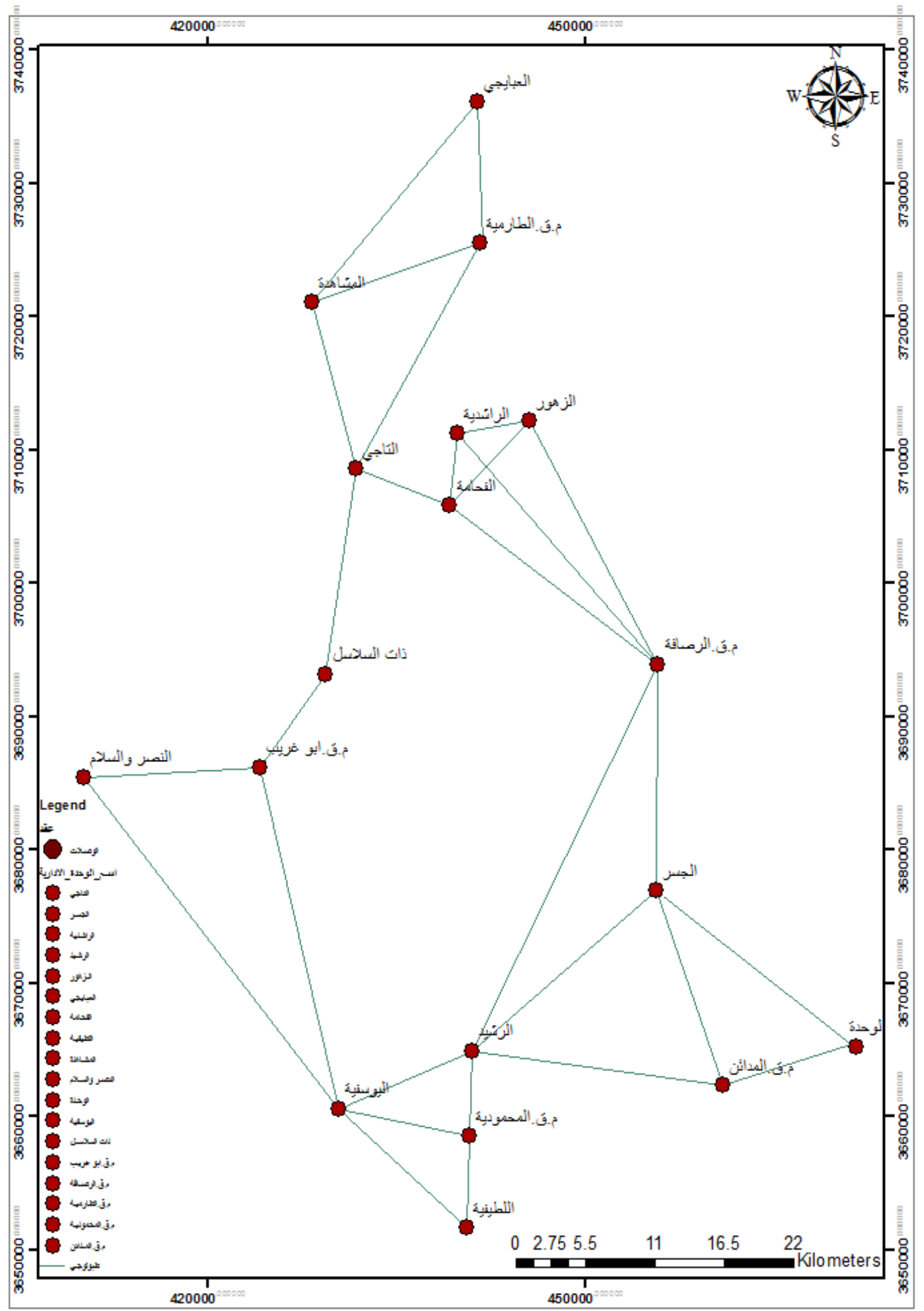




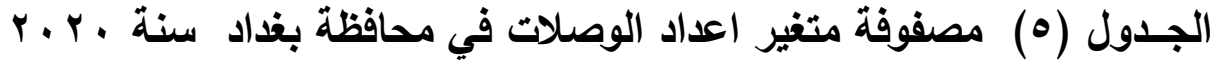

\begin{tabular}{|c|c|c|c|c|c|c|c|c|c|c|c|c|c|c|c|c|c|c|}
\hline ناحبة & ناحية المشاهدة & م.ق الطارية & والسـلام & غربب & ناحية & اللطية & ناحبة & م.ق. المحمودية & ناحية الوحدة & ناحية & م.ق. & م.ق .قضاء & ناحية & |ناحية ذات & ناحية & الفحامية & ناحية الراشدبة & المراكز الحضرية \\
\hline$\varepsilon$ & $r$ & $r$ & $\varepsilon$ & $\varepsilon$ & $r$ & $\varepsilon$ & $r$ & $r$ & $\mu$ & $r$ & $r$ & 1 & $r$ & $r$ & 1 & 1 & & ناحية الر اشدية \\
\hline$\varepsilon$ & $\mu$ & $\mu$ & $\varepsilon$ & $\varepsilon$ & $r$ & $\xi$ & $\mu$ & $r$ & $\mu$ & $r$ & $r$ & 1 & $r$ & $r$ & & 1 & 1 & ناحية الزهور \\
\hline$\mu$ & $r$ & $r$ & $r$ & 1 & $\mu$ & $\mu$ & $r$ & $\mu$ & 0 & $\varepsilon$ & $\varepsilon$ & $\mu$ & 1 & & $\mu$ & $r$ & $\mu$ & ناحية ذات السلاسل \\
\hline$r$ & 1 & 1 & $r$ & $r$ & $\mu$ & $\varepsilon$ & $r$ & $\varepsilon$ & $\varepsilon$ & $\mu$ & $\varepsilon$ & $r$ & & 1 & $r$ & 1 & $r$ & ناحية التاجي \\
\hline 7 & 0 & 0 & $\mu$ & $\mu$ & 1 & $\mu$ & $r$ & r & 1 & 1 & & $r$ & $\varepsilon$ & $\varepsilon$ & $\mu$ & $r$ & $\mu$ & م.ق. المدائن \\
\hline 0 & $\varepsilon$ & $\varepsilon$ & $\mu$ & $\mu$ & 1 & $\mu$ & $r$ & $r$ & 1 & & 1 & 1 & $\mu$ & $\varepsilon$ & $r$ & $r$ & $r$ & ناحية الجسر \\
\hline 7 & 0 & 0 & $\varepsilon$ & $\varepsilon$ & $r$ & $\varepsilon$ & $\mu$ & $\mu$ & & 1 & 1 & $r$ & $\varepsilon$ & 0 & $\mu$ & $r$ & $r$ & ناحية الوحدة \\
\hline 7 & 0 & 0 & $r$ & $r$ & 1 & 1 & 1 & & $\mu$ & $r$ & $r$ & $r$ & $\varepsilon$ & $\mu$ & $\mu$ & $r$ & $\mu$ & م.ق. المحمودية \\
\hline 0 & $\varepsilon$ & $\varepsilon$ & 1 & 1 & 1 & 1 & & 1 & $\mu$ & $r$ & $r$ & $r$ & $\mu$ & $r$ & $\mu$ & $r$ & $\mu$ & ناحية اليوسفية \\
\hline 7 & 0 & 0 & r & r & $r$ & & 1 & 1 & $\varepsilon$ & $\mu$ & $r$ & $r$ & $\varepsilon$ & $r$ & $\varepsilon$ & $\varepsilon$ & $\varepsilon$ & ناحية اللطيفية \\
\hline 0 & $\varepsilon$ & $\varepsilon$ & r & r & & $r$ & 1 & 1 & r & 1 & 1 & 1 & r & r & r & r & r & ناحية الرشبد \\
\hline$\varepsilon$ & $r$ & $\mu$ & 1 & & r & $r$ & 1 & r & $\varepsilon$ & $r$ & $r$ & $r$ & r & 1 & $\varepsilon$ & $r$ & $\varepsilon$ & م.ق. ابو غريب \\
\hline 0 & $\varepsilon$ & $\varepsilon$ & & 1 & $r$ & $r$ & 1 & r & $\varepsilon$ & $r$ & $r$ & $r$ & $\mu$ & $r$ & $\varepsilon$ & $\varepsilon$ & $\varepsilon$ & النصر و السلام \\
\hline 1 & 1 & & $\varepsilon$ & $r$ & $\varepsilon$ & 0 & $\varepsilon$ & 0 & 0 & $\varepsilon$ & 0 & $r$ & 1 & $r$ & $r$ & $r$ & $r$ & م.ق.الطار مية \\
\hline \multirow[t]{2}{*}{1} & & 1 & $\varepsilon$ & $r$ & $\varepsilon$ & 0 & $\varepsilon$ & 0 & 0 & $\varepsilon$ & 0 & $r$ & 1 & $r$ & $r$ & $r$ & $r$ & ناحية المشاهدة \\
\hline & 1 & 1 & 0 & $\varepsilon$ & 0 & 7 & 0 & 7 & 7 & 0 & 7 & $\varepsilon$ & $r$ & $r$ & $\varepsilon$ & $r$ & $\varepsilon$ & ناحية العبايجي \\
\hline$V \cdot$ & 00 & 00 & 01 & $\leqslant 0$ & rh & 07 & $\S 1$ & $\Sigma \wedge$ & 01 & $\varepsilon r$ & 01 & re & $\sum r$ & $\leqslant 7$ & $\sum 7$ & $\varepsilon$ & $\sum 7$ & المجموع \\
\hline $1 \leq$ & 11 & 11 & 1. & V & r & ir & $\varepsilon$ & 9 & س & 7 & 1. & 1 & 0 & $\wedge$ & $\Lambda$ & r r r r & $\wedge$ & الرتبة المرجحة \\
\hline
\end{tabular}


أ. مؤثر بيتا Beta Index:

وهو من ابسط المقاييس التي يمكن من خلالها قياس درجة النزابط بين شبكة النقل وفق لقانق

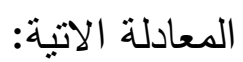

$$
\begin{aligned}
& \text { مؤشر بيتا = عدد العقدات الوصلات } \\
& 1.0=\frac{\Delta V .}{\text { OTr }}=
\end{aligned}
$$

تتراوح قيمة مؤشر بيتا بين صفر وواحد عدد صحيح ، ويعني قيمة المؤشر الصفر ان

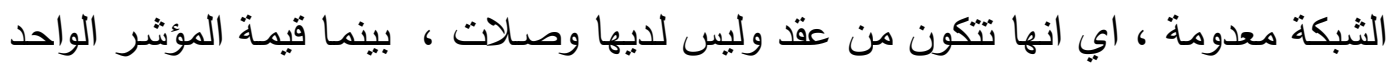

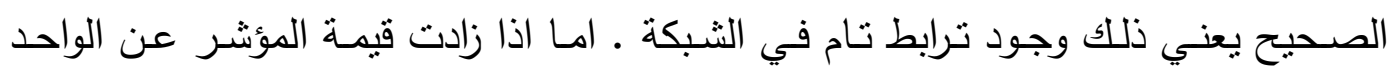

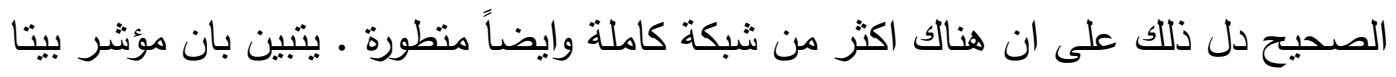

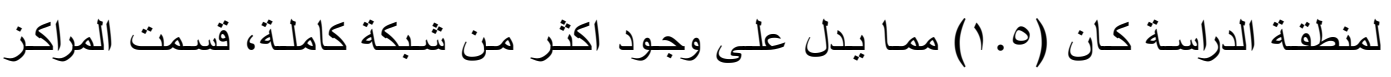

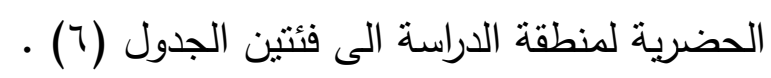

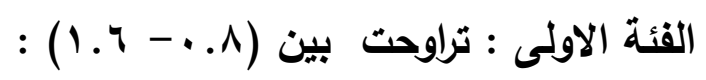

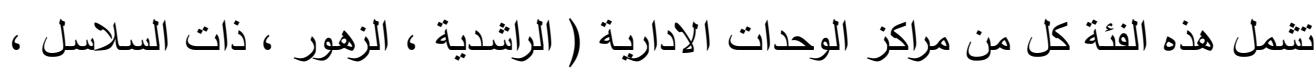

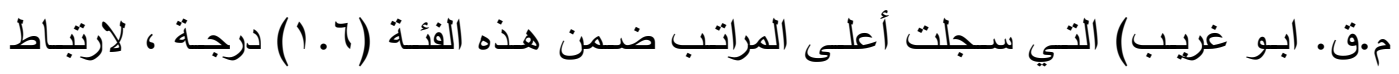

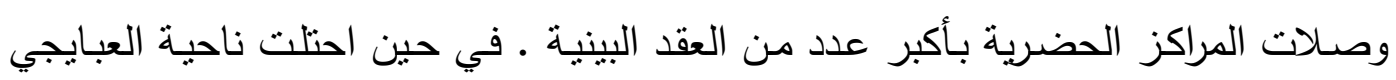

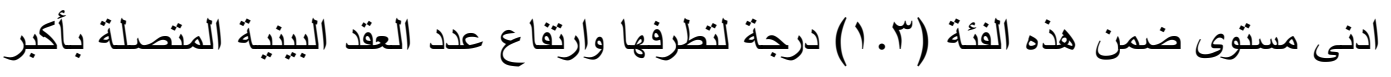

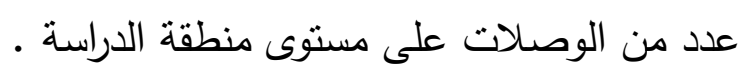

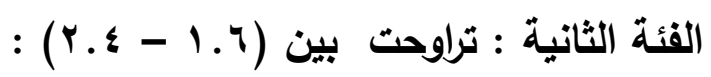

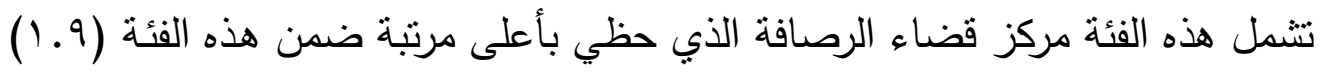

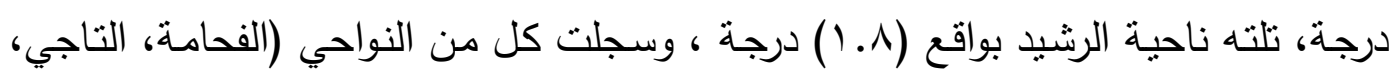

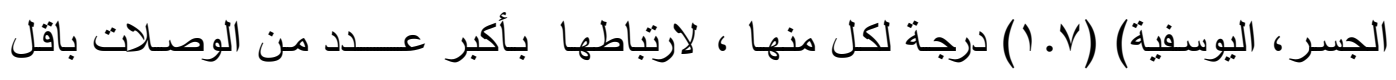
عــدد من العقــــ البينية على مستوى منطقة الدراســة.

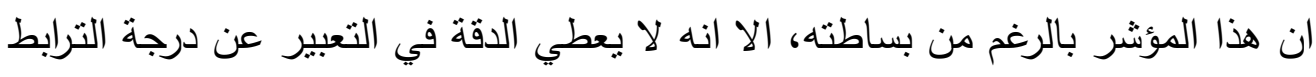

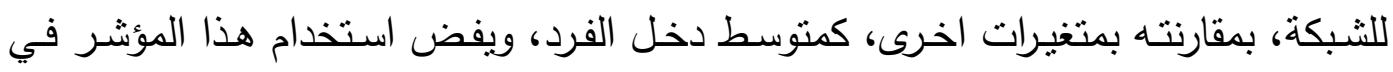

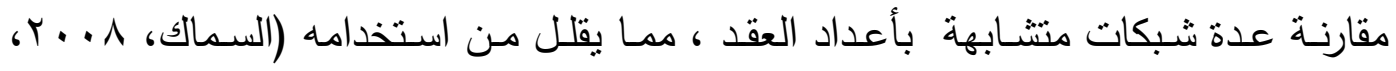
صفحة ع (1) (1).

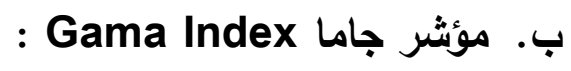
يعد من المقاييس المهمة لقياس درجة الترابط شبكة الطرق بين المراكز الحضرية ، ويأخذ بعين الاعنبار عدد الوصلات المكن وجودها في الثبكة وفق الصيغة النالية : 


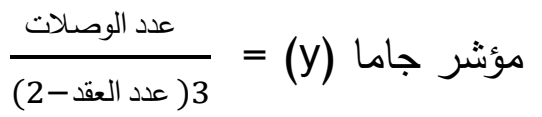

ويمكن وصف هذا المؤشر رقمياً مدى ترابط الثبكة ، اذا تتراوح قيمته بين الصفر

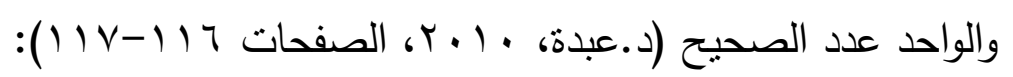

الفئة الاولى : فعندما تكون قيمة المؤشر صفر فان ذللك يعني بان الثبكة عديمة الترابط

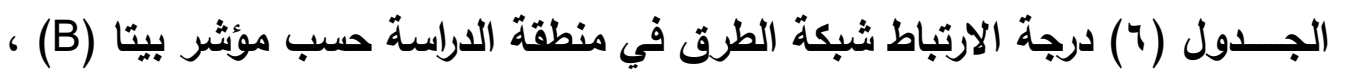

\begin{tabular}{|c|c|c|c|c|c|c|}
\hline Aؤشرة & المؤشر قيمة & قائشة & عدد العقد & الوصلات & المراكز الحضرية & ت \\
\hline 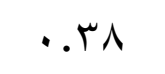 &. $.0 \mathrm{~V}$ & 1.7 & rq & $\leqslant 7$ & ناحية الراثندية & 1 \\
\hline .0 & 每. & $1 . V$ & rr & $\varepsilon$. & ناحية الفحامة & r \\
\hline..$\top \wedge$ & $.0 \mathrm{~V}$ & 1.7 & rq & $\leq 7$ & ناحية الزهور & $r$ \\
\hline..$\mu \Lambda$ &. $.0 \mathrm{~V}$ & 1.7 & rq & $\leqslant 7$ & ناحية ذات السلاسل & $\varepsilon$ \\
\hline$\cdot . \leqslant 0$ &. .71 & $1 . v$ & ro & $\varepsilon r$ & ناحية التاجي & 0 \\
\hline. .7 &. .79 & 1.9 & $r$. & $r v$ & م.ق. الرصافة & 1 \\
\hline.$\mu_{1}$ & .04 & 1.0 & $r \varepsilon$ & 01 & م.ق. المدائن & v \\
\hline..$\leqslant \Gamma$ & .7 & $1 . V$ & rT & $\varepsilon r$ & ناحية الجسر & $\wedge$ \\
\hline. ro & .0 & $1 . \varepsilon$ & $\varepsilon 1$ & $0 \wedge$ & ناحية الوحدة & 9 \\
\hline. ro &. .00 & 1.0 & r & $\leqslant \wedge$ & م.ق. المحمودية & 1. \\
\hline$\cdot . \leqslant V$ &. $.7 r$ & $1 . V$ & $r \varepsilon$ & $\varepsilon$ & ناحية اليوسفية & 11 \\
\hline..$Y 4$ & .0 & $1 . \varepsilon$ & rq & 07 & ناحية اللطيفية & ir \\
\hline .07 &. $.7 V$ & 1.1 & r) & ra & ناحية الرشيد & ir \\
\hline..$r \Lambda$ &. .01 & 1.7 & rA & «o & م.ق. ابو غريب & $1 \varepsilon$ \\
\hline.$\Gamma^{\top}$ & $.0 \%$ & 1.0 & $r \varepsilon$ & 01 & النصر والسلام & 10 \\
\hline.$r V$ &. .01 & $1 . \varepsilon$ & rᄉ & 00 & م.ق.الطارمية & 17 \\
\hline..$r V$ &. .01 & $1 . \varepsilon$ & rA & 00 & ناحية المشاهدة & iv \\
\hline. .19 &..$\leqslant 7$ & $1 . r$ & or & $v \cdot$ & ناحية العبايجي & 11 \\
\hline..$r \Lambda$ & $.0 Y$ & 1.0 & 074 & $14 \Lambda$ & \multicolumn{2}{|l|}{ المجموع الكلي } \\
\hline
\end{tabular}

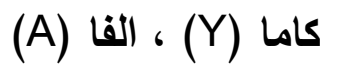




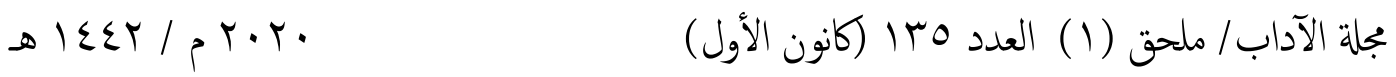

الفئة الثانية: اما اذا كانت قيمة المؤشر اقل من الواحد عدد صحيح فانه يعبر عن وجود ترابط ضعيف في الثبكة .

الفئة الثالثة: بينما اذا كانت قيمة المؤشر واحد عدد صحيح فانه يعبر عن وجود نرابط في

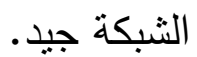

الفئة الرابعـة: واخيراً اذ كانت قيمة المؤشر اكثر من الواحد عدد صحيح فانه يعبر عن وجود ترابط قوي في الثبكة (جيد جداً) •

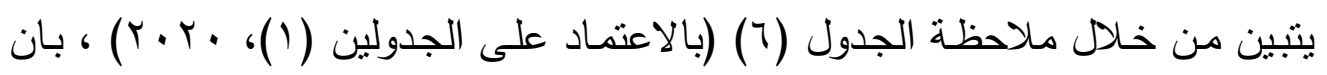
المراكز الحضرية في منطقة الدراسة تقع جميعها ضمن الفئة الثانية لمؤشر جاما ، اذ بلغت

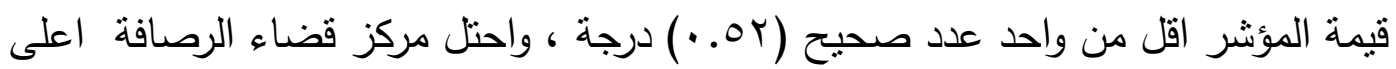
نسبة في منطقة الدراسة (79. . ) درجة ، تلته ناحية الرشيد بالمرتبة الثانية (VV. . ) درجة ،

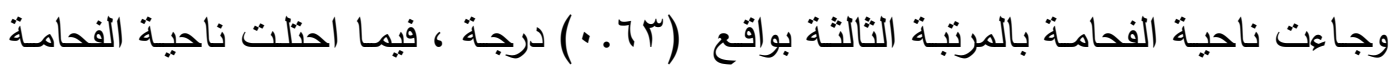

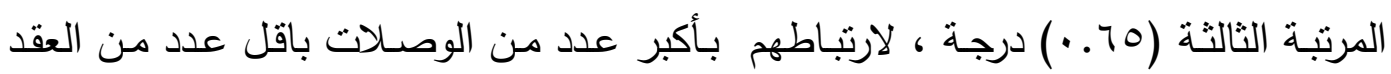

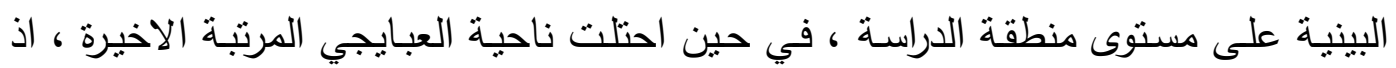

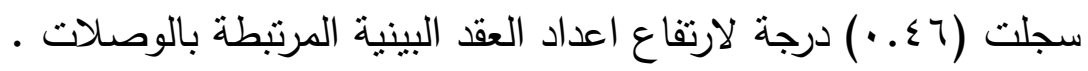

\section{ج. Aوئثر الفا Alfa Index}

وهو من المقاييس المهـة في قياس درجة الترابط لثبكة النقل، وخاصـة المعقدة بين

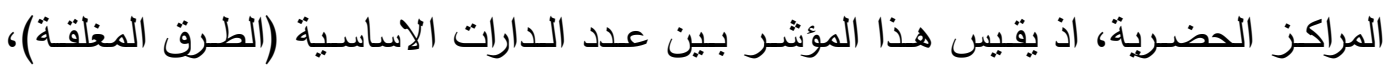
واقصى عدد لها من خلال ايجاد العلاقة بين اجزاء الطرق المغلقة واقصى عدد ممكن لها

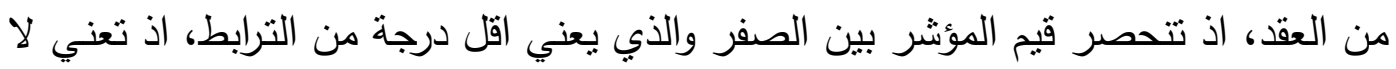

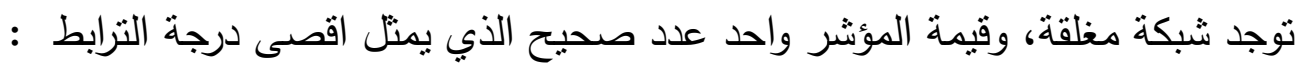

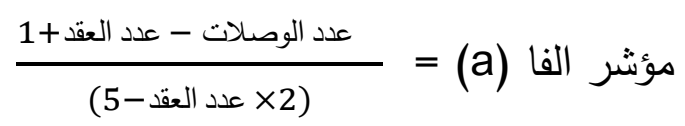

ويمكن وصف هذا المؤشر رقمياً مدى ترابط الثبكة اذا تتراوح قيمته بين الصفر والواحد

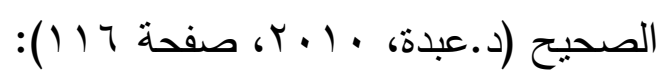

الفئة الاولى : عندما تكون قيمة المؤشر صفر فانه بعني بان الثبكة عديمة الترابط .

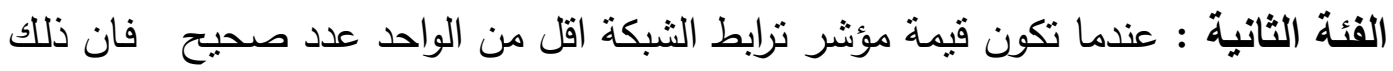
يعبر عن ضعف الترابط بين اجزاء الثبكة . الفئة الثالثة : بينما اذا كانت قيمة المؤشر واحد عدد صحيح فانه يعبر عن وجود ترابط

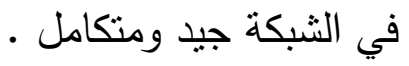


يتضـح مـن خـلال ملاحظـة الجدول (؟)، ان المراكز الحضـرية في منطقة الدراسـة

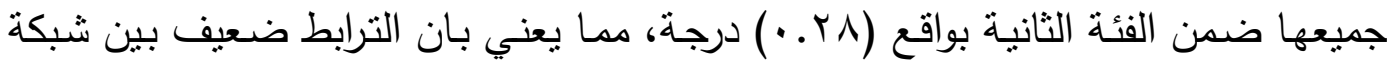
الطرق في منطقة الدراسة، موزعة بين المراكز الحضرية بدرجات متفاوتة ، اذ احتلت ناحية مركز قضاء الرصافة على اعلى مرنبة بلغت (T. ·) درجة، لزيادة عدد الوصلات المتصلة بالعقد البينية، واحتلت ناحية الرشيد المرتبة الثانية اذ سجلت (ها هـ • درجة، واحتلت ناحية

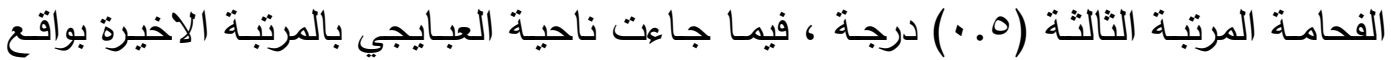
(9 ( . ) درجة، بسبب زيادة عدد العقد البينية المتصلة بالوصلات.

: Abler Index دوشر ابلر

يعد مؤشر ابلر من المقاييس المهــة في قياس درجـة الاتصـال لثبكة الطرق بين المراكز الحضرية لمنطقة الدراسة، اذ يمكن قياس درجة الاتصال من خلال معادلات ثلاثة ضمن هذا المؤشر : - المرن الفئة الاولى: الصلة الادنى تعني ان درجة الاتصال بين اجزاء الثبكة تتراوح (الصفر واقل الفئـة الثانيـة: الصلة الاعلى تعني ان درجة الاتصـال بين اجزاء الثبكة تساوي (1) عدد الفئة الثالثة: الصلة الحالية لثبكة الطرق تعني مقارنة بين شبكة الطرق الادنى وبين شبكة الطرق الاعلى، اذا كانت القيمة المؤشرة قريبة الصلة من الحد الادنى يعني ذلك انها شبكة

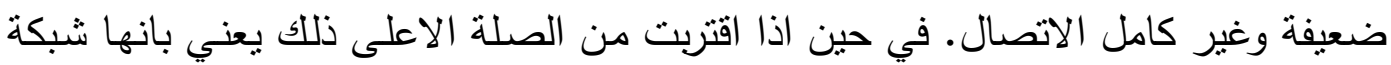
جيدة وعالية الكفاءة من حيث الاتصال بين المراكز الحضرية. ويمكن فياس درجة الاتصال

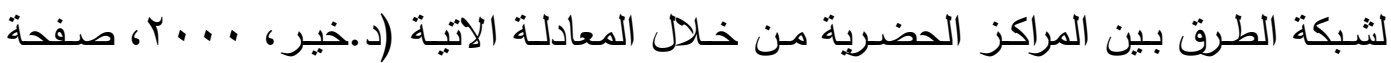
$:(\leqslant$ ro

$$
\begin{aligned}
& \frac{\mathrm{N}-1}{\left(N^{2}-N\right) / 2}= \\
& \frac{W}{\left(N^{2}-N\right) / 2}=
\end{aligned}
$$

ويمثل اعلى صلة = ا عدد صحيح (عدد نسبي ثابت لا يتغير) = N = W

تبين مـن خـلال معطيـات الجدول (V)، بـان منوسط المؤشـر الصـلة الادنى لمنطقة

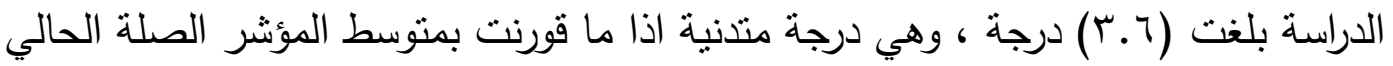
لمنطقة الدراسـة، والتي بلغت (0.0) درجـة دل ذللك على ان الثبكة ضعيفة وغير كاملـة الاتصال، وهناك تباين بين المراكز الحضرية في منطقة الدراسة ضمن فئة الحد الادنى اقل 
من (1) عدد صحيح ، اذ حظي مركز قضاء الرصافة بالمرتبة الاولى ، بواقع (1. (·) درجة

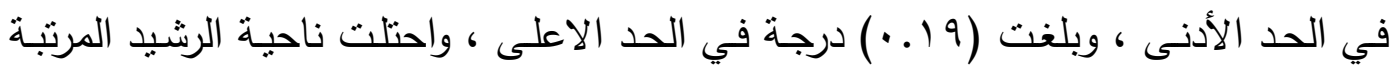

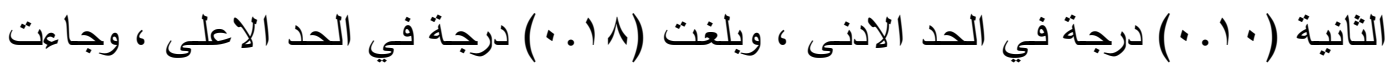

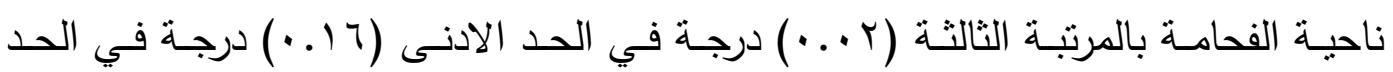

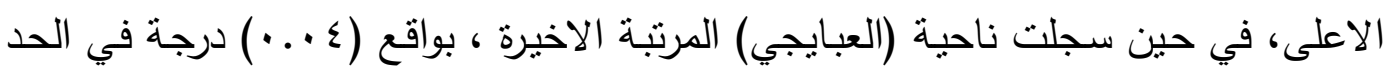

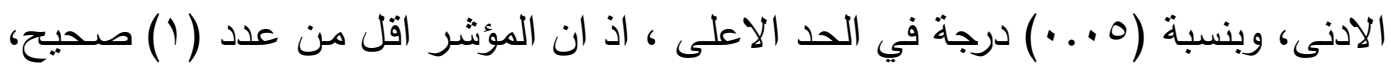

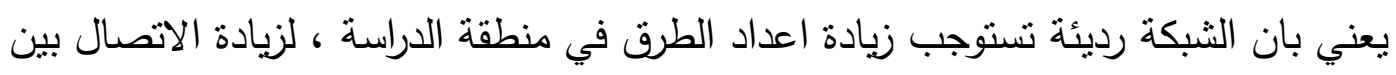
اجزاء شبكة الطرق، والاقتراب من الدد الاعلى للشبكة ، بتخطبط طرق جديدة مكملة للطرق الرابطة بين مراكزها، كالطرق الحلقية الرابطة .

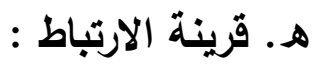

يعد مؤشر قرينة الارتباط من المؤشرات المهمة لقياس كفاءة شبكة الطرق، بالاستخلال في تماثل شبكة الطرق المعبدة بين المراكز الحضرية لمنطقة الدراسة، وتحديد اتصاليتها،

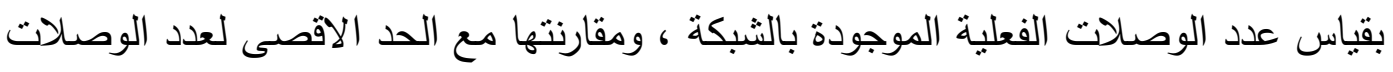
الممكنة في الثبكة، ويمكن حساب قياس قرينة الارتباط من خلال المعادلة الاتية : $\frac{W}{\left(W^{2}-W\right) 0.5}=$ قرينة الارتباط

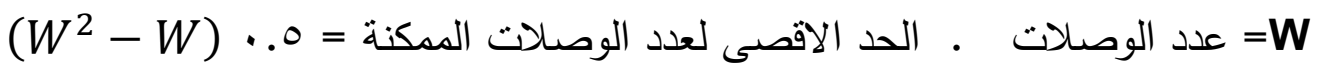

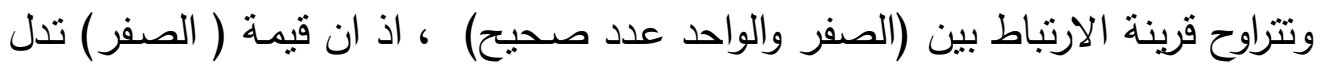

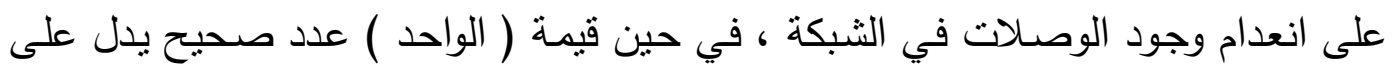

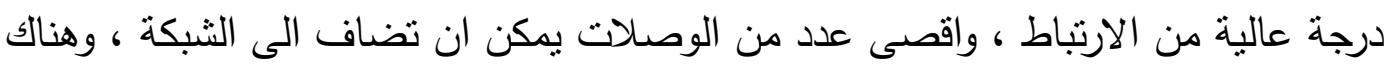
ثلاثة حالات من درجات الارتباط يمكن ان ثقاس بها شبكة الطرق لمنطقة الدراسة هي :

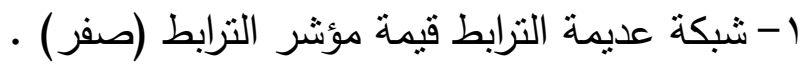

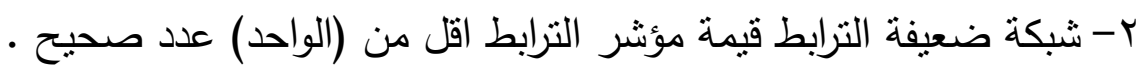

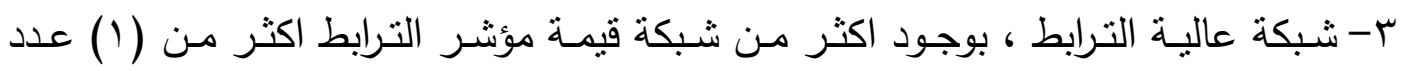
صحيح

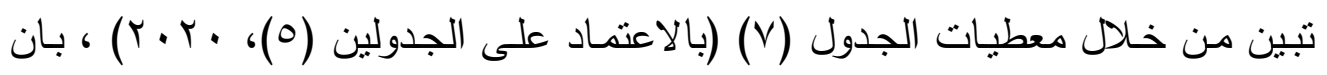

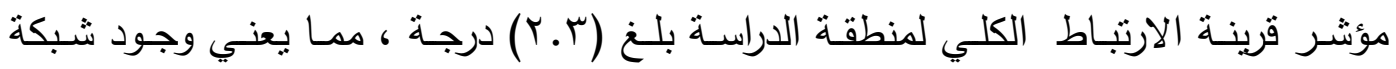

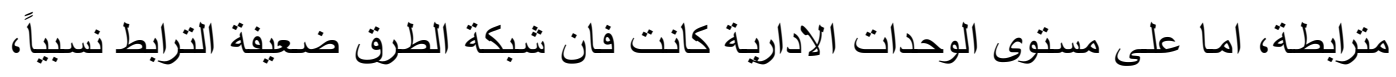

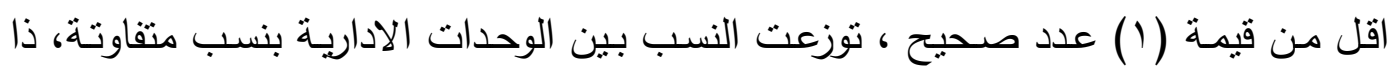

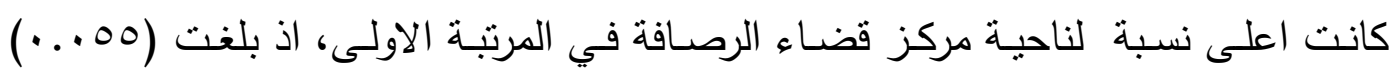

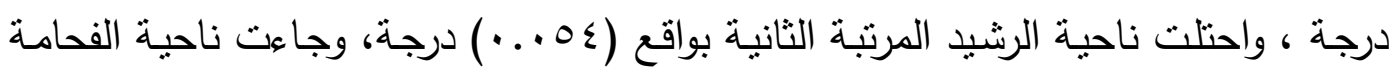


بالمرتبـة الثالثة اذ سـجلت (01. . •) درجـة ، في حين جـاءت ناحيـة (العبايجي) بالمرتبـة الاخيرة، بواقع (^^ . . •) درجة ـ مما يبين ضعف ترابط الثبكة بين المراكز ، الامر الذي بتطلب تحديث المخططات الاساسية للطرق المجره الجـدول (V) درجة الارتباط شبكة الطرق في منطقة الدراسة حسب مؤشر ابلر

\begin{tabular}{|c|c|c|c|c|c|c|}
\hline مؤشر الارتباط & الصالية & صلة ادنى & عدد العقد & عدد & المراكز الحضرية & $ت$ \\
\hline$\cdot . \cdot \leq \varepsilon$ &. .11 & $\cdots v$ & rq & $\leqslant 7$ & ناحية الراشدية & 1 \\
\hline. .01 &. .17 & $\ldots r$ & r & $\varepsilon$. & ناحية الفحامة & r \\
\hline 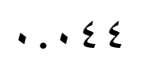 &. .11 & $\cdot . v$ & rq & $\leqslant 7$ & ناحية الزهور & r \\
\hline$\cdot . \cdot \leq \varepsilon$ &. .11 & $\ldots v$ & $r q$ & $\varepsilon 7$ & ناحية ذات السلاسل & $\varepsilon$ \\
\hline$\cdot . \leqslant \Lambda$ & $. .1 \leq$ & $\cdots 1$ & ro & $\varepsilon r$ & ناحية التاجي & 0 \\
\hline .000 & .19 & .1 & $r$. & rv & م.ق. الرصافة & 7 \\
\hline$\cdot . \varepsilon$ &. .9 &. .7 & $r \varepsilon$ & 01 & م.ق. المدائن & $\checkmark$ \\
\hline$\cdot . \leqslant V V$ & rו & $\cdots \wedge$ & $r y$ & $\varepsilon r$ & ناحية الجسر & $\wedge$ \\
\hline ס ... & $\ldots v$ &. .0 & $\leqslant$ & $0 \wedge$ & ناحية الوحدة & 9 \\
\hline$\cdot . \leqslant Y$ & .1 & $\cdot .7$ & I & $\varepsilon \wedge$ & م.ق. المحمودية & 1. \\
\hline$\ldots 0$ & .10 &..$\wedge$ & $r \leq$ & $\leqslant 1$ & ناحية اليوسفية & 11 \\
\hline דוr. & $\ldots \wedge$ & $\ldots 0$ & rq & 07 & ناحية اللطيفية & IT \\
\hline$. .0 \leqslant$ &. .11 &. .1 & YI & r & ناحية الرشيد & $i$ \\
\hline$\cdots \leqslant 0$ & .14 & $\cdots v$ & $r \wedge$ & $\leqslant 0$ & م.ق. ابو غريب & $1 \varepsilon$ \\
\hline$\cdot . \varepsilon$ &. .9 &. .7 & re & 01 & النصر والسلام & 10 \\
\hline ..rV & $\ldots .1$ &. .0 & r & 00 & م.ق .الطارمية & 17 \\
\hline..$r V$ & $\ldots .1$ & $\ldots 0$ & rᄉ & 00 & ناحية المشاهدة & IV \\
\hline$\cdots r \wedge$ & $\ldots 0$ & $\cdots \varepsilon$ & ror & $V$. & ناحية العبايجي & 11 \\
\hline T.r & 0.0 & $r .7$ & $07 Y$ & ヘ૫^ & \multicolumn{2}{|l|}{ المجموع الكلي } \\
\hline
\end{tabular}




\section{توصل البحث الى مجموعة من النتائج تتمثل بالاتي:}

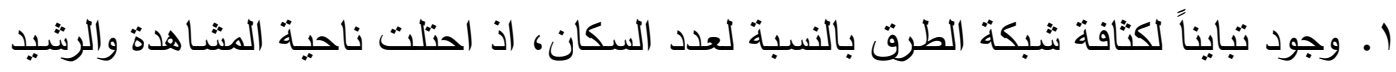

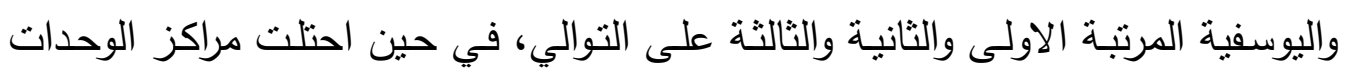
الادارية (الراندية وذات السلاسل ومركز قضاء الددائن) المرتبة الاخيرة .

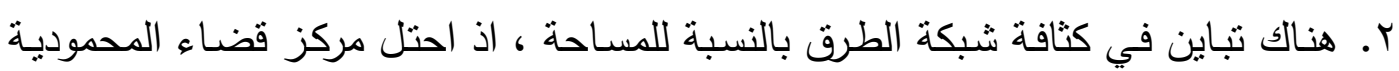

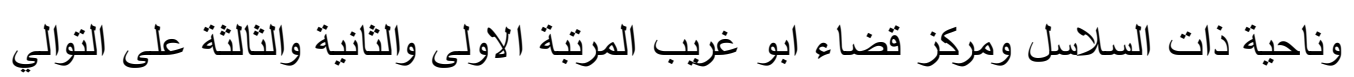
، فيما جاءت ناحية الوحدة بالمرتبة الاخيرة .

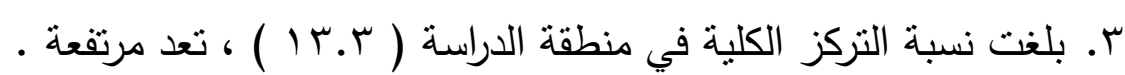

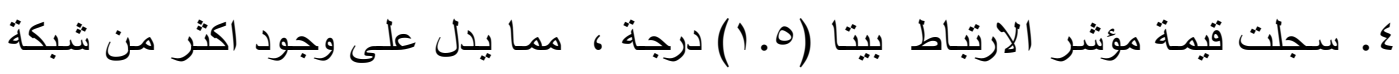
كاملة .

0. بلغت قيمة مؤشر الارتباط جاما (Yo. •) درجة ، مما يدل على وجود ترابط ضعيف في

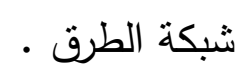

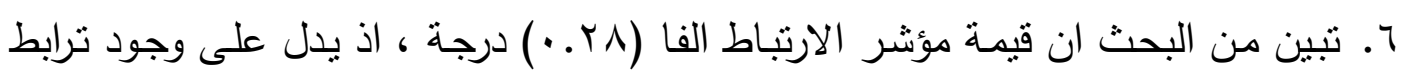
ضعيف في الثبكة.

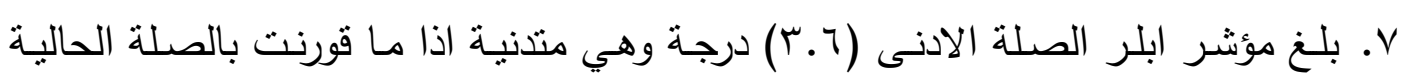

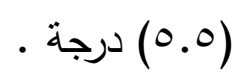

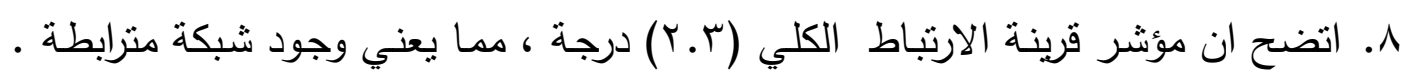

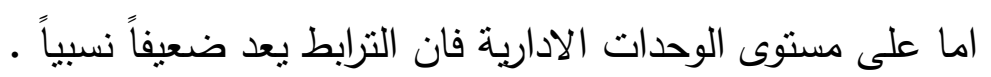

المصادر العربية عل ملئئ

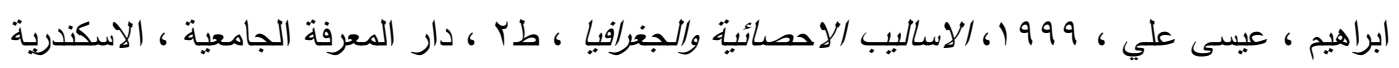

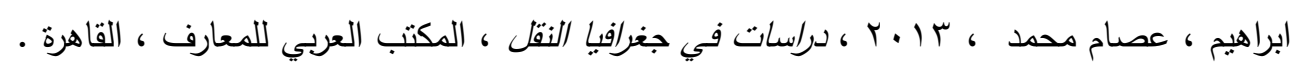

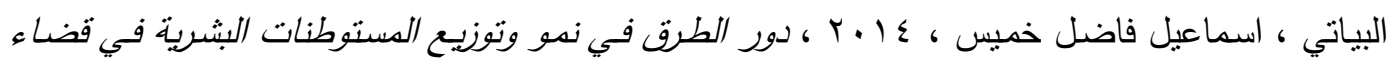

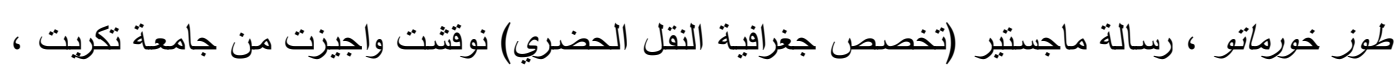

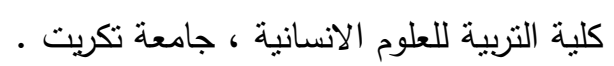

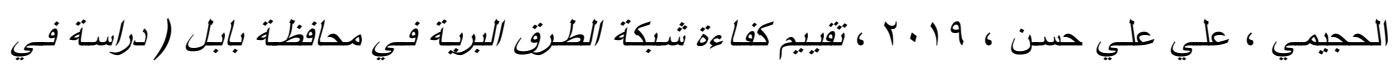

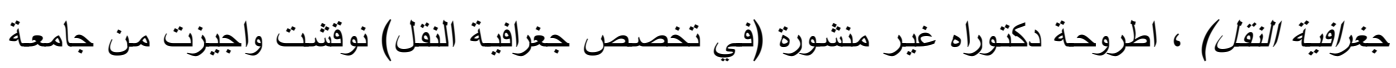
البصرة ، كلية التربية للعلوم الانسانية ، جامعة البصرة.

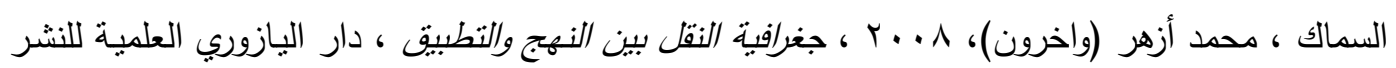

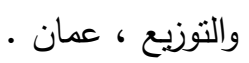
جمهوريـة العراق ، وزارة الاسكان والاعمـار والبلديات والاشــال العامـة ، مديريـة الطرق والجسور ، قسم

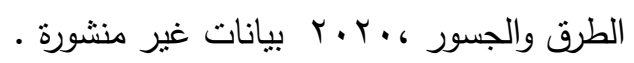




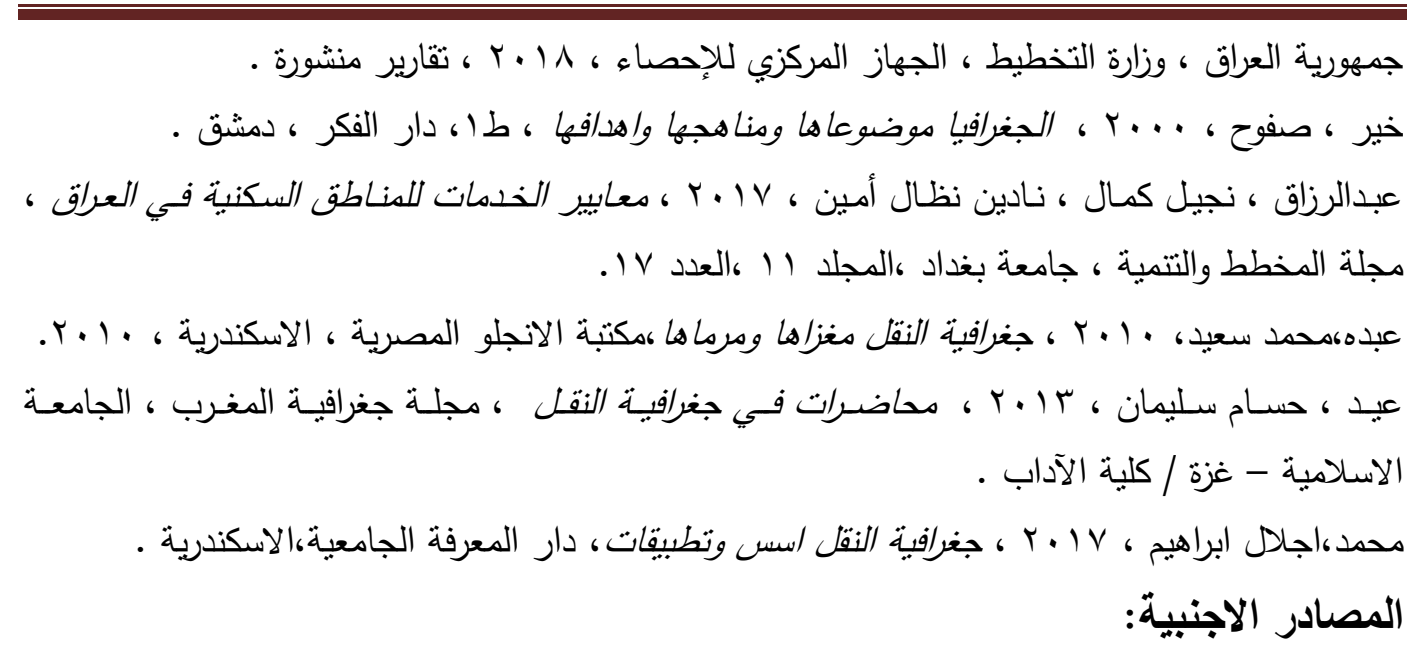

Adams, 19 १^، p., Network Topologies and Virtual Place, Annals of the A. A. G. , Vol. 88, No. 1.

\section{References:}

Ibrahim, Issa Ali، 1999 , Statistical Methods and Geography, 2nd floor, University Knowledge House, Alexandria.

Ibrahim, Essam Mohamed, $Y_{\bullet}, \uparrow$ ، Studies in Transport, Arab Knowledge Bureau, Cairo.

Al-Bayati, Ismail Fadel Khamis, 2014 , The Role of Roads in the Growth and Distribution of Human Resources in the Tuz Khurmato District, Master Thesis (Urban Geography Specialization) was discussed and approved by Tikrit University, College of Education for Humanities, Tikrit University.

Al-Hujaimi, Ali Ali Hassan, 2019 , Assessment of the Efficiency of the Land Road Network in Babil Governorate (A Study in the Geography of Transport), an unpublished doctoral thesis (in the field of transport geography) was discussed and approved by the University of Basra, College of Education for Humanities, University of Basra.

Al-Sammak, Muhammad Azhar , 2008 , (and others), Geography of Transport between Approach and Implementation, Al-Yazouri Scientific Publishing and Distribution House, Amman.

Republic of Iraq, Ministry of Housing, Construction, Municipalities, and Public Works, Directorate of Roads and Bridges, Department of Roads and Bridges, 2019 , unpublished data.

Republic of Iraq, Ministry of Planning, Central Statistical Organization, 2018, published reports.

Khair, Sufouh, 2000 , Geography, its Subject, Approaches, and Goals, 1st Floor, Dar Al-Fikr, Damascus.

Abdul-Razzaq, Najil Kamal, Nadine Nazal Amin, 2007, Standards of Services for Residential Areas in Iraq, The Planner and Development Magazine, University of Baghdad, Volume 11, No. 17.

Abdo, Mohamed Said, 2010 , Geography of Transport Meaning and Goal, The Anglo Egyptian Library, Alexandria.

Eid, Hossam Suleiman, 2013 , Lectures on the Geography of Transport, Geography of Morocco, Islamic University - Gaza / College of Arts.

Mohamed, Ajal Ibrahim, 2017 , Geography of Transport, Bases and Applications, University Knowledge House, Alexandria.

Adams , 1998 , p., Network Topologies and Virtual Place, Annals of the A. A. G. , Vol. 88, No. 1, March 1998. 
Evaluating the efficiency of the transportation network between urban centers in Baghdad Governorate

Researcher. Bashar Jebur Ali basharj154@gmail.com

Asst.Prof.Dr. Abdulrahman Jery Al-Howaider rahmanja19@yahoo.com

\title{
Summary
}

\author{
Basra University / College of Education for Humanities
}

The research aims to demonstrate the efficiency of the road network linking urban centers in Baghdad governorate, through a quantitative assessment of the reality of the road network, to provide the required services in a smooth and high efficiency for their users, as they represent the arteries of movement between urban centers, so it required the use of a number of approved metrics and indicators to give an indication of Efficiency of roads and to achieve the goal of the research, as the research dealt with nodes, connections and lengths of roads spatially distributed at the level of the Baghdad governorate, as each node is connected to direct and indirect connections and different lengths. The study applied quantitative indicators in measuring the efficiency of the road network linking the nodes, The road density scale for the population and the area has found a difference in the degree of density between the administrative units according to the population numbers, the area of their administrative units and the length of the roads, while the road concentration index for the study area reached a high total concentration rate of (13.3\%), indicating that the length of the roads in it You need to redistribute within the total area, and the beta correlation index reached that of the study area according to the index value that reached (1.5) with more than one complete network, and the gamma correlation index according to the index value (0.52) reached a degree less than one integer with weak correlation in the network Roads, The alpha correlation index in the study area according to the index value $(0.28)$ reached a score less than one integer with a weak correlation between parts of the network, and the average correlation index Abler reached that the degree of the lowest correlation index for the study area reached (3.6) degrees, which is a low score if compared to an average The index of the current relevance of the study area reached (5.5) degrees, indicating that the network is weak and incomplete communication according to the indicator, and the index of correlation reached for the study area according to the value of the total indicator reached (2.3) degrees, which means that there is a connected network at the governorate level, either at the level of Administrative units The value of the indicator was less than (1) an integer, indicating that the road network has a relatively weak correlation, and by applying quantitative indicators it is possible to reach knowledge of the strength and weakness of the road network, and choose successful solutions for it.

key words :1. Connections 2. Contract 3. Efficiency.4. Road network 